\title{
Cerenkov radiation from moving straight strings
}

\author{
D.V. Gal'tsov*, E.Yu. Melkumova* and K. Salehi* \\ ${ }^{*}$ Department of Physics, Moscow State University,119899, Moscow, Russia
}

\begin{abstract}
We study Cerenkov radiation from moving straight strings which glisse with respect to each other in such a way that the projected intersection point moves faster than light. To calculate this effect we develop classical perturbation theory for the system of Nambu-Goto strings interacting with dilaton, two-form and gravity. In the first order one encounters divergent self-action terms which are eliminated by classical renormalization of the string tension. Cerenkov radiation arises in the second order. It is generated by an effective source which contains contributions localized on the strings world-sheets and bulk contributions quadratic in the first order fields. In the ultra-relativistic limit radiation exhibits angular peaking on the Cerenkov cone in the forward direction of the fast string in the rest frame of another. The radiation spectrum then extends up to high frequencies proportional to square of the Lorentz-factor of the relative velocity. Gravitational radiation is absent since the $1+2$ space-time transverse to the straight string does not allow for gravitons. A rough estimate of the Cerenkov radiation in the cosmological cosmic strings network is presented.
\end{abstract}

PACS numbers: 11.27.+d, 98.80.Cq, 98.80.-k, 95.30.Sf

\section{INTRODUCTION}

During past few years the hypothesis of cosmic strings received new impetus from superstring theory. Although perturbative superstrings in ten dimensions are too heavy to be admitted as cosmic strings, it was realized that there are various possibilities for geometry of string compactifications to accomodate four-dimensional strings with much lower tensions. Copious production of cosmic strings is typical [1, 2] for the brane inflation scenario [3], in which the period of inflation is associated with the collision of branes. This scenario provides an acceptable model of inflation and predicts creation of cosmic superstrings consistent with the current CMB data. These strings typically have lower tensions than the usual GUT cosmic strings and thus they are not the main players in the formation of cosmic structures, but their observational signatures could provide a direct confirmation of the string theory. This stimulated a detailed study of creation and evolution of the cosmic superstring network within the KKLMMT model [4], racetrack inflation [5] and other particular scenarios [6, 7, 8] (for recent review see [9, 10, 11, 12, 13]). One particular feature of the warped IIB compactifications involved in these considerations is prediction of two types of cosmic strings: F-strings (fundamental) and D-strings (Dirichlet) with different tensions $\mu_{F}, \mu_{D} \sqrt{14}$, 15, 16, 17]. F and $\mathrm{D}$ strings may also form the so-called $p, q-$ composites [18] and provide for triple junctions [19] with new exotic observational predictions. Typical values of the dimensionless parameter $G \mu$ are in the interval $\left(10^{-11}-10^{-7}\right)$. Another new feature is that cosmic superstrings generically have lower reconnection probabilities lying in the range $P \sim\left(10^{-3}-1\right)$. This changes cosmological evolution of the string network [20, 21, 22, 23, 24] leading in particular to enhancement of the fraction of straight strings.

Both the string network evolution and their possible observational signatures crucially depend on radiation processes. In fact, it was recognized long ago that oscillating loops of cosmic strings generate large output of gravitational waves $[25,26,27,28]$ at the level accessible for current and future detectors. Global strings produce massless axions $[28,[29,30,31,32,33$ ] which become massive at a later stage of expansion, creating an observational constraint on the axion mass. In the models containing the massless dilaton (like cosmic superstrings), the dilaton radiation from strings may also constrain the string tension parameter $G \mu[34, \underline{35}, \underline{36}]$.

The main mechanisms of radiation in the string network which have been explored so far were radiation from smoothly oscillating string loops and radiation from kinks and cusps formed on them. It was tacitly assumed that straight unexcited long strings do not radiate. Meanwhile, interaction of long strings via massless fields gives rise to another radiation mechanism of Cerenkov nature. When two straight non-excited Nambu-Goto strings interacting at a distance via dilaton, two-form and gravity glisse with respect to each other, they get deformed in the vicinity of the point of their minimal separation. This point can propagate with a faster-than light velocity, provided the inclination angle between the strings is sufficiently small. (For the strictly parallel strings this velocity is infinite for any relative string speed.) In this case, the propagating deformation together with the associated field tensions become the source of Cerenkov radiation, which is the effect of the second order in interaction of the string with massless fields. This mechanism was suggested in [37] for gravitationally interacting strings, but it turned out that, although the effect is kinematically allowed, the corresponding amplitude is zero on the mass-shell of the graviton. The reason is that the $1+2$ space-time orthogonal to the straight string does note allow for gravitons. (However, gravitons are produced at quantum level in the strings recombination and annihilation [38].) But Cerenkov mechanism works for other possible 
string massless excitations leading, e.g., to electromagnetic radiation from superconducting strings [39] or emission of axions. The latter efect was recently studied in detail in the flat space-time [40]. It was found that, although being of the second order in the axion coupling constant, it still gives a considerable contribution into the total cosmological axion production by the string network.

Cerenkov radiation from straight strings is similar to bremsstrahlung of point charges in electrodynamics. Moreover, the system of parallel strings interacting via a two-form field in D space-time dimensions is exactly equivalent to the system of point charges interacting with the vector field in (D-1)-dimensional space-time. A distinction of the Cerenkov mechanism of dissipation in the cosmic string network from conventional radiation via formation of loops can be understood as follows. Formation of loops from initially disconnected straight strings is effected via direct contact interaction of intersecting strings. In our case the long-range interaction of strings via massless fields which can be potentially radiated underlies the formation of the superluminal radiation source. Cerenkov radiation is a higher order effect and thus potentially smaller. But it works for a wider set of initial data in the string network (non-zero impact parameters of colliding strings), than that corresponding to intercommuting strings. Also,as we will show here, Cerenkov radiation is strongly enhanced in the case of relativistic velocities. So it can still be non-small in the cosmological setting. The detailed cosmological applications remain outside the scope of the present paper, but we give the rough estimates of the dilaton and two-form field backgrounds generated via Cerenkov mechanism in the evolving string network.

In this paper we consider Cerenkov radiation of moving straight strings interacting with three massless fields: dilaton, antisymmetric second rank tensor (NS-NS or RR two-forms) and gravity. To facilitate construction of the solution of the coupled system of the Nambu-Goto and the field equations in the second order we introduce a diagrammatic representation similar to Feynmann graphs. In the first order approximation one encounters divergencies which are cured by classical renormalization of the string tension. Then the effective sources of the dilaton and two-form radiation are constructed and the radiation rates are calculated.

The plan of the paper is as follows. In Sec. 2 we give the general setting of the problem and introduce graphic representation for classical vertices corresponding to interactions of strings with dilaton, two-form and gravity and to interactions between the fields. Sec. 3 contains formulation of the perturbation approach and an explicit construction of the second order equations. In Sec. 4 we reproduce in our framework the main features of classical renormalization for strings (in the lowest order of perturbation theory): elimination of scalar and two-form divergences via renormalization of the string tension, and the absence of gravitational divergency. In Sec. 5 the first order deformations of the string world-sheets due to interaction via the dilaton, two-form and linearized gravity are considered. The main calculation is presented in Sec. 6 where we construct radiation generating currents by computing the source terms in the wave equations of the second order. These include the world-sheet local terms and the bulk terms which correspond to multiple second order graphs including all relevant elementary vertices. Radiation rates for the dilaton and the twoform fields are computed in the Sec. 7 and analyzed in detail in the case of ultrarelativistic velocities. Finally, in Sec. 8 we present rough cosmological estimates and summarize our results in Sec. 9.

\section{ACTION AND EQUATIONS OF MOTION}

We consider a pair $n=1,2$ of straight Nambu-Goto strings described by the world-sheets

$$
x^{\mu}=X_{n}^{\mu}\left(\sigma_{n}^{a}\right), \quad \mu=0,1,2,3, \quad \sigma_{a}=(\tau, \sigma), \quad a=0,1 .
$$

Strings interact via the gravitational $g_{\mu \nu} \equiv \eta_{\mu \nu}+h_{\mu \nu}$, the dilaton $\phi(x)$ and the two-form (axion) $B_{\mu \nu}(x)$. Using the Polyakov form for the string action, we present the total action in the form:

$$
\begin{aligned}
S & =-\sum \int\left\{\frac{\mu}{2} X_{a}^{\mu} X_{b}^{\nu} g_{\mu \nu} \gamma^{a b} \sqrt{-\gamma} \mathrm{e}^{2 \alpha \phi}+2 \pi f X_{a}^{\mu} X_{b}^{\nu} \epsilon^{a b} B_{\mu \nu}\right\} d^{2} \sigma+ \\
& +\int\left\{2 \partial_{\mu} \phi \partial_{\nu} \phi g_{\mu \nu}+\frac{1}{6} H_{\mu \nu \rho} H^{\mu \nu \rho} \mathrm{e}^{-4 \alpha \phi}-\frac{R}{16 \pi G}\right\} \sqrt{-g} d^{4} x,
\end{aligned}
$$

where the sum is taken over $n=1,2$ (the corresponding index is omitted for brevity). Our signature choice is mostly minus for the space-time metric, and $(+,-)$ for the world-sheets. The Levi-Cività symbol is $\epsilon^{01}=-\epsilon^{10}=1, \gamma_{a b}$ is the metric on the world-sheet, and the lower Latin indices mean partial derivatives with respect to the world-sheet coordinates $X_{a}^{\mu} \equiv \partial_{a} X^{\mu}$. The action contains four parameters: the string tension $\mu$, the Newton constant $G$, the dilaton coupling $\alpha$ and the two-form coupling $f$. The antisymmetric three-form field strength is defined as

$$
H_{\mu \nu \lambda}=\partial_{\mu} B_{\nu \lambda}+\partial_{\nu} B_{\lambda \mu}+\partial_{\lambda} B_{\mu \nu} .
$$


Variation of the action (2) with respect to $X^{\mu}$ leads to the equations of motion for strings

$$
\partial_{a}\left(\mu X_{b}^{\nu} g_{\mu \nu} \gamma^{a b} \sqrt{-\gamma} \mathrm{e}^{2 \alpha \phi}+4 \pi f X_{b}^{\nu} \epsilon^{a b} B_{\mu \nu}\right)=X_{a}^{\lambda} X_{b}^{\nu} \partial_{\mu}\left(\frac{\mu}{2} g_{\lambda \nu} \gamma^{a b} \sqrt{-\gamma} \mathrm{e}^{2 \alpha \phi}+2 \pi f \epsilon^{a b} B_{\lambda \nu}\right)
$$

The coordinate derivatives on the right hand side apply to the metric, dilaton and two-form fields, their values are taken on the world sheet. The derivatives with respect to the world-sheet coordinates $\sigma^{a}$ on the left hand side apply both to the world-sheet quantities $X^{\nu}\left(\sigma^{a}\right), \gamma_{a b}$ and to the metric, dilaton and two-form fields, e.g., for the metric

$$
\partial_{a} g_{\mu \nu}=X_{a}^{\lambda} \partial_{\lambda} g_{\mu \nu}
$$

and similarly for $\phi, B_{\mu \nu}$. In this formalism $\gamma_{a b}$ is an independent variable; variation of the action with respect to $\gamma_{a b}$ gives the constraint equation

$$
\left(X_{a}^{\mu} X_{b}^{\nu}-\frac{1}{2} \gamma_{a b} \gamma^{c d} X_{c}^{\mu} X_{d}^{\nu}\right) g_{\mu \nu}=0
$$

whose solution defines $\gamma_{a b}$ as the induced metric on the world-sheet:

$$
\gamma_{a b}=X_{a}^{\mu} X_{b}^{\nu} g_{\mu \nu}
$$

Consider now the field equations. Variation over $\phi$ gives the dilaton equation

$$
\partial_{\mu}\left(g^{\mu \nu} \partial_{\nu} \phi \sqrt{-g}\right)+\frac{\alpha}{6} H^{2} \mathrm{e}^{-4 \alpha \phi}=-\sum \frac{\mu \alpha}{4} \int X_{a}^{\mu} X_{b}^{\nu} g_{\mu \nu} \gamma^{a b} \sqrt{-\gamma} \mathrm{e}^{2 \alpha \phi} \delta^{4}(x-X(\sigma, \tau)) d^{2} \sigma
$$

where the sum in the source term is taken over the contribution of two stings. The equation for the two-form field reads:

$$
\partial_{\mu}\left(H^{\mu \nu \lambda} \mathrm{e}^{-4 \alpha \phi} \sqrt{-g}\right)=-\sum 2 \pi f \int X_{a}^{\nu} X_{b}^{\lambda} \epsilon^{a b} \delta^{4}(x-X(\sigma, \tau)) d^{2} \sigma .
$$

We also have the Bianchi identity

$$
\nabla_{[\mu} H_{\alpha \beta \gamma]}=0
$$

where alternation over indices has to be performed and the derivative can be equivalently treated as a covariant derivation with respect to $g_{\mu \nu}$ or a partial derivative.

Finally, for the metric we have the Einstein equations

$$
R_{\mu \nu}-\frac{1}{2} g_{\mu \nu} R=8 \pi G\left(\stackrel{\phi}{T_{\mu \nu}}+\stackrel{B}{T} \mu \nu+\stackrel{s t}{T} \mu \nu\right)
$$

where the source terms read

$$
\begin{aligned}
& \stackrel{s t}{T}^{\mu \nu}=\sum \mu \int X_{a}^{\mu} X_{b}^{\nu} \gamma^{a b} \sqrt{-\gamma} \mathrm{e}^{2 \alpha \phi} \frac{\delta^{4}(x-X(\sigma, \tau))}{\sqrt{-g}} d^{2} \sigma, \\
& \stackrel{\phi}{T}^{\mu \nu}=4\left(\partial^{\mu} \phi \partial^{\nu} \phi-\frac{1}{2} g^{\mu \nu}(\nabla \phi)^{2}\right), \\
& \stackrel{B}{T}^{\mu \nu}=\left(H_{\alpha \beta}^{\mu} H^{\nu \alpha \beta}-\frac{1}{6} H^{2} g^{\mu \nu}\right) \mathrm{e}^{-4 \alpha \phi} .
\end{aligned}
$$

The total system of equations consists of two equations for stings of the type (41) and the field equations (8, 9, 11). It describes classically in a self-consistent way motion of the strings with account for their interaction via the dilaton, two-form and gravitational fields as well as evolution of the generated fields. This system can be solved iteratively in terms of the coupling constants $\alpha, f, G$. To facilitate this construction it is convenient to introduce a classical analog of the Feynmann graphs, denoting string by bald solid line, the dilaton by this solid line, the two-form by dashed and graviton by wavy lines (Fig. 1). Simple analysis of the equations reveals that we have vertices involving linear and non-linear string-field interactions, as well as three-leg and multi-leg interactions of fields between themselves. 

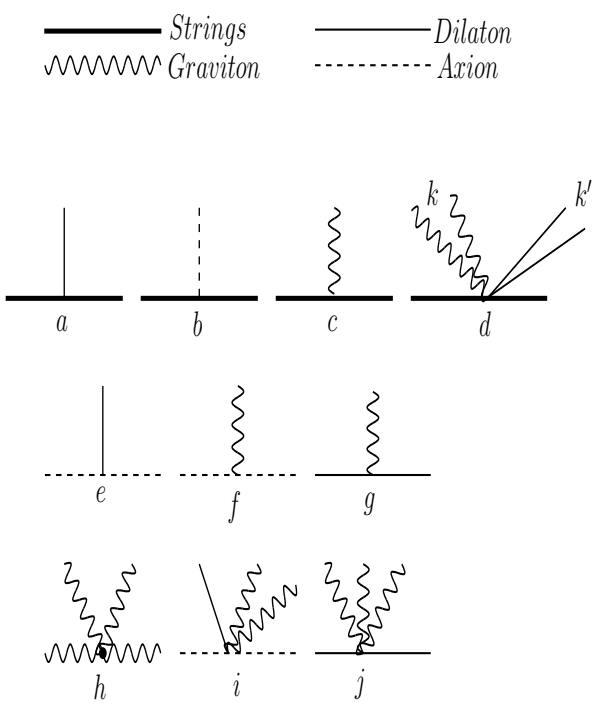

Рис. 1: Vertices associated with the action (2): graphs $a, b, c$ are string-dilaton, string-two-form and string-graviton vertices of the lowest order; graph $d$ depicts higher-order string-dilaton-graviton vertices (for all $k \geq 1, k^{\prime} \geq 1$ ); graphs $e, f, g$ are lowest order field interactions present in the action (2), and graphs $e, f, g$ are multi-graviton vertices accompanying the lowest order ones.

\section{PERTURBATION THEORY}

We construct an iterative solution of the coupled string-field system expanding the string world-sheet mapping functions $X_{n}^{\mu}\left(\sigma^{a}\right), n=1,2$, and the field variables in terms of the coupling constants $\alpha, f, G$ :

$$
\begin{aligned}
& X^{\mu}=\stackrel{0}{X}^{\mu}+\stackrel{1}{X}^{\mu}+\ldots, \\
& \phi=\stackrel{1}{\phi}+\stackrel{2}{\phi}+\ldots, \\
& B_{\mu \nu}=\stackrel{1}{B}_{\mu \nu}+\stackrel{2}{B}_{\mu \nu}+\ldots, \\
& h_{\mu \nu}=\stackrel{1}{h}_{\mu \nu}+\stackrel{2}{h}_{\mu \nu}+\ldots
\end{aligned}
$$

Here the expansions of $X_{n}^{\mu}$ start from the zero order, while those of the field variables - from the first order terms, that is we assume that there are no background dilaton, two-form or gravitational fields. Zero order mapping functions describe the straight infinite uniformly moving strings

$$
\stackrel{0}{X}_{n}^{\mu}=d_{n}^{\mu}+u_{n}^{\mu} \tau+\Sigma_{n}^{\mu} \sigma .
$$

Here $\Sigma_{n}^{\mu}$ is the unit spacelike constant vectors along the strings, and $u_{n}^{\mu}$ are the unit timelike constant vectors - fourvelocities of the strings. The corresponding three-dimensional velocities are orthogonal to the strings. The constant vectors $d_{n}^{\mu}$ can be regarded as impact parameters for two strings with respect to the chosen frame. In the zero order the space-time metric is flat, and the corresponding world-sheet metrics are also Minkowskian $\eta_{a b}=\operatorname{diag}(1,-1)$ in view of the normalization assumed

$$
\left(\Sigma_{n} \Sigma_{n}\right)=\eta_{\mu \nu} \Sigma_{n}^{\mu} \Sigma_{n}^{\nu}=-1, \quad\left(u_{n} u_{n}\right)=1, \quad\left(\Sigma_{n} u_{n}\right)=0 .
$$

It is convenient the choose the Lorentz frame in which the first string is at rest and is stretched along the z-axis:

$$
u_{1}^{\mu}=[1,0,0,0], \quad \Sigma_{1}^{\mu}=[0,0,0,1] .
$$

The second string is assumed to move in the plane $x^{2}, x^{3}$ with the velocity $v$ orthogonal to the string itself:

$$
u_{2}^{\mu}=\gamma[1,0,-v \cos \alpha, v \sin \alpha], \quad \Sigma_{2}^{\mu}=[0,0, \sin \alpha, \cos \alpha],
$$

where $\gamma=\left(1-v^{2}\right)^{-1 / 2}$. In such a configuration the strings never intersect each other remaining always in the parallel planes. Apart from the orthogonality conditions (17), four other scalar products are

$$
\left(u_{1}, u_{2}\right)=\gamma, \quad\left(\Sigma_{1}, \Sigma_{2}\right)=-\cos \alpha, \quad\left(u_{1}, \Sigma_{2}\right)=0, \quad\left(u_{2}, \Sigma_{1}\right)=-v \gamma \sin \alpha
$$


note that $u_{1}$ and $\Sigma_{2}$ are orthogonal. We also choose both impact parameters $d_{n}^{\mu}$ to be orthogonal to $u_{n}^{\mu}$ and $\Sigma_{n}^{\mu}$ and aligned with the axis $x^{1}$, the distance between the planes being $d=d_{2}-d_{1}$. The angle of inclination $\alpha$ of the second string with respect to the first one can be written in a Lorentz-invariant form

$$
\alpha=\arccos \left(-\Sigma_{1} \Sigma_{2}\right)
$$

Similarly, the invariant expression for the relative velocity of the strings is

$$
v=\left(1-\left(u_{1} u_{2}\right)^{-2}\right)^{\frac{1}{2}}
$$

With this parametrization of the unperturbed world-sheets, the projected intersection point (the point of the minimal separation between the strings) moves with the velocity

$$
v_{p}=\frac{v}{\sin \alpha}=\left(u_{1} u_{2}\right)^{-1}\left(\frac{\left(u_{1} u_{2}\right)^{2}-1}{1-\left(\Sigma_{1} \Sigma_{2}\right)^{2}}\right)^{\frac{1}{2}}
$$

along the $x^{3}$-axis. This motion is not associated with propagation of any signal, so the velocity $v_{p}$ may be arbitrary, in particular, superluminal $v_{p}>1$. The case of parallel strings corresponds to $v_{p}=\infty$.

Note that the above introduced parameters (22) 21 ) are not invariant under reparameterizations of the world-sheets [40]. The quantity which is invariant under the volume preserving reparameterizations is

$$
\kappa=\operatorname{det}\left(X_{1 a}^{\mu} X_{2 b}^{\nu} \eta_{\mu \nu}\right)=\gamma \cos \alpha
$$

The superluminal regime corresponds to $\kappa>1$.

The expansions (15) are substituted into the system of equations (4, 8, 9, 11) which has to be solved iteratively. The zero order differential operator in the dilaton equation (4) is the flat-space D'Alembert operator $\square=-\eta^{\mu \nu} \partial_{\mu} \partial_{\nu}$. Similarly, choosing the Lorentz gauge for the two-from and the metric perturbations

$$
\partial_{\mu} B^{\mu \nu}=0, \quad \partial_{\mu} \psi^{\mu \nu}=0, \quad \psi^{\mu \nu}=h^{\mu \nu}-\frac{1}{2} \eta^{\mu \nu} h,
$$

where $h=h_{\mu}^{\mu}$, we get the linear D'Alembert equations for the first order two-form and the gravitational field as well. Due to linearity of the field equations, the first order dilaton, two-form and metric perturbations can be presented as the sums of the separate contributions due to two strings

$$
\begin{aligned}
\stackrel{1}{\phi} & =\stackrel{1}{\phi_{1}+{ }^{\phi}}, \\
\stackrel{1}{B}^{\mu \nu} & =\stackrel{1}{B_{1}^{\mu \nu}}+\stackrel{1}{B}_{2}^{\mu \nu}, \\
\stackrel{1}{h}^{\mu \nu} & =\stackrel{1}{h}_{1}^{\mu \nu}+\stackrel{1}{h}_{2}^{\mu \nu} .
\end{aligned}
$$

Here each term with $n=1,2$ satisfies the individual D'Alembert equation with the source labelled by the same index $n$ :

$$
\begin{aligned}
\square \stackrel{1}{\phi}_{n} & =4 \pi \stackrel{0}{J}_{n}, \\
\square \stackrel{1}{B}_{n}^{\mu \nu} & =4 \pi \stackrel{0}{J}_{n}^{\mu \nu}, \\
\square \stackrel{1}{h} \mu \nu & =4 \pi \stackrel{0}{\tau}_{n}^{\mu \nu} .
\end{aligned}
$$

The coupling constants are included into the source terms, while zero indices in the sources indicate that they are computed using the zero order approximations for the strings mapping functions. The source terms thus read

$$
\begin{aligned}
& \stackrel{0}{J_{n}}=\frac{\alpha \mu}{8 \pi} \int \delta^{4}\left(x-\stackrel{0}{X_{n}}(\tau, \sigma)\right) d^{2} \sigma \\
& \stackrel{0}{J}_{n}^{\mu \nu}=\frac{f}{2} \int V_{n}^{\mu \nu} \delta^{4}(x-\stackrel{0}{X}(\tau, \sigma)) d^{2} \sigma, \\
& \stackrel{0}{\tau}_{n}^{\mu \nu}=4 G \mu_{n} \int W_{n}^{\mu \nu} \delta^{4}(x-\stackrel{0}{X}(\tau, \sigma)) d^{2} \sigma,
\end{aligned}
$$


where the following antisymmetric and symmetric tensors are introduced

$$
\begin{aligned}
V_{n}^{\mu \nu} & =\epsilon^{a b} \stackrel{0}{X_{a n}^{\mu}} \stackrel{0}{X_{b n}^{\nu}}=u_{n}^{\mu} \Sigma_{n}^{\nu}-u_{n}^{\nu} \Sigma_{n}^{\mu}, \\
U_{n}^{\mu \nu} & =\eta^{a b} \stackrel{0}{X_{a n}^{\mu}} \stackrel{0}{X_{b n}^{\nu}}=u_{n}^{\mu} u_{n}^{\nu}-\Sigma_{n}^{\mu} \Sigma_{n}^{\nu}, \quad U_{n}^{\mu \nu} \eta_{\mu \nu}=U_{n}=2, \\
W_{n}^{\mu \nu} & =U_{n}^{\mu \nu}-\frac{1}{2} \eta^{\mu \nu} U_{n} .
\end{aligned}
$$

Now consider the perturbations of the strings world-sheets induced metrics (index $n$ is omitted)

$$
\gamma_{a b}=\eta_{a b}+\stackrel{1}{\gamma}_{a b}
$$

where in the general gauge the first order correction reads

$$
\stackrel{1}{\gamma}_{a b}=\stackrel{0}{X} \stackrel{0}{a}^{X}{ }_{b}^{\nu} \stackrel{1}{h}_{\mu \nu}+2 \stackrel{0}{X_{a}^{\mu}} \stackrel{1}{X}{ }_{b}^{\nu} \eta_{\mu \nu}
$$

However, to be able to disentangle the higher order perturbed equations one has to get rid of the second term in (37) proportional to $\stackrel{1}{X}^{\nu}$. Using the space-time and the world-sheet diffeomorphism invariance, we can impose the gauge condition

$$
\stackrel{0}{X_{a}^{\mu}} \stackrel{1}{X}{ }_{b}^{\nu} \eta_{\mu \nu}=0
$$

in which case the perturbed induced metric will contain only the zero order string mapping functions:

$$
\stackrel{1}{\gamma}_{a b}=\stackrel{0}{X}_{a}^{\mu} \stackrel{0}{X}_{b}^{\nu} \stackrel{1}{h}_{\mu \nu}, \quad \stackrel{1}{\gamma}=U^{\mu \nu} \stackrel{1}{h}_{\mu \nu}
$$

The first order perturbations of the mapping functions $\stackrel{1}{X}^{\mu}$ describing deformations of the flat world-sheets satisfy the following equations following from the Eq.(4):

$$
\begin{gathered}
\mu \eta^{a b} \partial_{a} \stackrel{1}{X}_{b}^{\nu} \eta_{\mu \nu}+\mu \partial_{a}\left\{\stackrel{0}{X_{b}^{\nu}}\left[\left(\stackrel{1}{h}_{\mu \nu}+\frac{1}{2} \eta_{\mu \nu} \stackrel{1}{\gamma}\right) \eta^{a b}-\eta_{\mu \nu} \stackrel{1}{\gamma}^{a b}\right]+2 \alpha \eta_{\mu \nu} \eta^{a b} \phi\right\}+ \\
+4 \pi f \epsilon^{a b} \stackrel{0}{X}_{b}^{\nu} \partial_{a} \stackrel{1}{B}^{\mu \nu}-2 \alpha \mu \partial_{\mu} \stackrel{1}{\phi}-\frac{1}{2} U^{\lambda \nu} \partial_{\mu} \stackrel{1}{h}{ }_{\lambda \nu}-2 \pi f V^{\lambda \nu} \partial_{\mu} \stackrel{1}{B}_{\lambda \nu}=0
\end{gathered}
$$

where raising of the world-sheet indices is performed by the Minkowski metric:

$$
\stackrel{1}{\gamma}^{a b}=\stackrel{1}{\gamma}_{c d} \eta^{a c} \eta^{b d}, \quad \stackrel{1}{\gamma}=\stackrel{1}{\gamma}_{c d} \eta^{c d}
$$

Differentiating the field variables along the world-sheet according to the rules

$$
\partial_{a} \stackrel{1}{\phi}=\stackrel{0}{=}{ }^{\lambda} \partial_{\lambda} \stackrel{1}{\phi}, \quad \partial_{a} \stackrel{1}{B}{ }_{\mu \nu}=\stackrel{0}{=}{ }_{a}^{\lambda} \partial_{\lambda} \stackrel{1}{B}_{\mu \nu}, \quad \stackrel{1}{h} \mu \nu=\stackrel{0}{h^{\lambda}} \partial_{\lambda} \stackrel{1}{h}_{\mu \nu},
$$

we can rewrite the above equation as

$$
\mu \eta^{a b} \partial_{a} \partial_{b} \stackrel{1}{X}^{\mu}=F_{(\phi)}^{\mu}+F_{(B)}^{\mu}+F_{(h)}^{\mu}
$$

where the forces due to dilaton, two-form and graviton are introduced:

$$
\begin{aligned}
& F_{(\phi)}^{\mu}=\alpha \mu\left(U \partial^{\mu} \stackrel{1}{\phi}-2 U^{\mu \nu} \partial_{\nu} \phi\right) \\
& F_{(B)}^{\mu}=2 \pi f V^{\nu \lambda} \stackrel{1}{H}_{\nu \lambda}^{\mu} \\
& F_{(h)}^{\mu}=\mu\left[\stackrel{1}{h \lambda \tau, \nu}^{\mu}\left(U^{\mu \lambda} U^{\nu \tau}-\frac{1}{2} U^{\mu \nu} U^{\lambda \tau}\right)-U_{\nu \lambda}\left(1^{\mu \nu, \lambda}-\frac{1}{2} h^{\nu \lambda, \mu}\right)\right]
\end{aligned}
$$

In these equations the indices labelling the strings are not shown, but it is understood that for each string we have to take into account at the right hand side both the self-force terms arising form the fields due to the same string, and the mutual interaction terms coming from the partner string. 
Consider now the second order field equations. Expanding the Eqs. (8)-(11) to the next order and imposing again the Lorentz gauge for the two-form and the linearized gravity one obtains the D'Alembert equations for the second order fields with the source terms involving contributions due to deformations of the strings world-sheets (local terms) as well as the quadratic combinations of the first order fields (bulk terms). The dilaton equation with account for (39) will read

$$
\begin{aligned}
\stackrel{2}{\phi}=4 \pi \stackrel{1}{J} & =\frac{\mu \alpha}{4} \sum \int\left[U^{\mu \nu}\left(\stackrel{1}{h}_{\mu \nu}+2 \eta_{\mu \nu} \alpha \stackrel{1}{\phi}\right)-4 \stackrel{1}{X}^{\mu} \partial_{\mu}\right] \delta^{4}[x-\stackrel{0}{X}(\tau, \sigma)] d^{2} \sigma+ \\
& +\frac{\alpha}{6} \stackrel{1}{H}^{\mu \nu \lambda} \stackrel{1}{H}_{\mu \nu \lambda}-\partial_{\mu}\left[\left(\stackrel{1}{h}^{\mu \nu}-\frac{1}{2} \eta^{\mu \nu} \stackrel{1}{h}\right) \partial_{\nu} \stackrel{1}{\phi}\right]
\end{aligned}
$$

where the partial derivative operator acts on the delta function. Note that the first order terms in the first line are multiplied by the dilaton coupling constant and thus give the same order quantities as the products of two first order field quantities.

The equation for the second order two-form field is:

$$
\begin{aligned}
& \stackrel{2}{B}^{\mu \nu}=4 \pi \stackrel{1}{J}^{\mu \nu}= \\
& =2 \pi f \sum \int\left(2 \epsilon^{a b} \stackrel{0}{X}_{a}^{[\mu} \stackrel{1}{X}_{b}^{\nu]} \delta^{4}[x-\stackrel{0}{X}(\tau, \sigma)]-V^{\mu \nu} \stackrel{1}{X}^{\lambda} \partial_{\lambda} \delta^{4}[x-\stackrel{0}{X}(\tau, \sigma)]\right) d^{2} \sigma+ \\
& +\partial_{\lambda}\left[\left(\frac{1}{2} \stackrel{1}{h}-4 \alpha \phi\right) \stackrel{1}{\phi} H^{\lambda \mu \nu}\right]
\end{aligned}
$$

where alternation over indices includes the factor $1 / 2$.

The situation is slightly more complicated for the graviton. To obtain an equation for the second order gravitational perturbation one has to take into account the quadratic terms in the Einstein equations. In the gauge

$$
\partial_{\mu} \psi^{\mu \nu}=0
$$

one has the following expansion of the Einstein tensor up to the second order:

$$
R_{\mu \nu}-\frac{1}{2} g_{\mu \nu} R=\frac{1}{2} \square \psi_{\mu \nu}+\frac{1}{2} S_{\mu \nu}\left(h_{\lambda \tau}\right),
$$

where $\psi_{\mu \nu}$ contains the first and the second order quantities, and the quadratic term reads:

$$
\begin{aligned}
S_{\mu \nu} & =\left[\partial^{\beta} h_{\mu}^{\alpha}\left(\partial_{\alpha} h_{\nu \beta}-\partial_{b} h_{\nu \alpha}\right)-\frac{1}{2} \partial_{\mu} h^{\alpha \beta} \partial_{\nu} h_{\alpha \beta}-\frac{1}{2} h_{\mu \nu} \partial_{\alpha} \partial^{\alpha} h+\right. \\
& +h^{\alpha \beta}\left(\partial_{\nu} \partial_{\beta} h_{\mu \alpha}+\partial_{\mu} \partial_{\beta} h_{\nu \alpha}-\partial_{\mu} \partial_{\nu} h_{\alpha \beta}-\partial_{\alpha} \partial_{\beta} h_{\mu \nu}\right)+ \\
& \left.+\frac{1}{2} \eta_{\mu \nu}\left(2 h^{\alpha \beta} \partial^{\lambda} \partial_{\lambda} h_{\alpha \beta}-\partial_{\lambda} h_{\alpha \beta} \partial_{\beta} h^{\alpha \lambda}+\frac{3}{2} \partial_{\lambda} h_{\alpha \beta} \partial^{\lambda} h^{\alpha \beta}\right)\right] .
\end{aligned}
$$

Extracting the second order terms we obtain from the Eq.(11):

$$
\square \stackrel{2}{\psi}_{\mu \nu}=16 \pi G \stackrel{1}{\tau}_{\mu \nu}
$$

where the source term reads:

$$
\begin{aligned}
\stackrel{1}{\tau}_{\mu \nu} & =\frac{\mu}{2} \sum \int\left(2 \stackrel{0}{X_{(\mu}^{a}} \stackrel{1}{X}_{\nu) a}-\stackrel{1}{X}{ }^{\lambda} \partial_{\lambda}\right) \delta^{4}(x-\stackrel{0}{X}(\tau, \sigma)) d^{2} \sigma+ \\
& +\frac{\mu}{4} \sum \int\left[\left(U_{\mu \nu} U^{\lambda \tau}-2 U_{\mu}^{\lambda} U_{\nu}^{\tau}\right) \stackrel{1}{h}{ }_{\lambda \tau}+U_{\mu \nu}(4 \alpha \stackrel{1}{\phi}-\stackrel{1}{h})\right] \delta^{4}(x-\stackrel{0}{X}(\tau, \sigma)) d^{2} \sigma+ \\
& +G^{-1} S_{\mu \nu}\left(\stackrel{1}{h}{ }_{\lambda \tau}\right)+2 \partial_{\mu} \stackrel{1}{\phi} \partial_{\nu} \stackrel{1}{\phi}-\eta_{\mu \nu} \partial_{\lambda} \stackrel{1}{\phi} \partial^{\lambda} \stackrel{1}{\phi}+\frac{1}{2} \stackrel{1}{H} \mu \stackrel{1}{H} \stackrel{1}{H}_{\nu}^{\lambda \tau}-\frac{1}{12} \eta_{\mu \nu} \stackrel{1}{H} \stackrel{1}{\nu \lambda \tau}^{\nu \lambda \tau}
\end{aligned}
$$

All source terms in the second order field equations have similar structure. Note the presence of the derivatives from delta functions in the strings world-sheet contributions.

It is worth noting that the second order dilaton, two-form and gravity fields already can not be presented as a sum of terms due to separate strings, but rather look as generated by the collective sources. These sources contain contributions not only from the perturbed world-sheets, but also the bulk contributions which are not associated with separate strings. As we will see later, these sources may have superluminal nature, in which case the Cerenkov radiation will appear. 


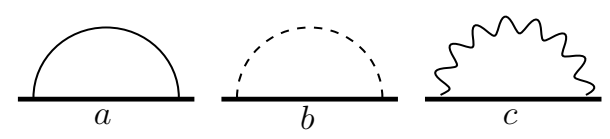

Рис. 2: Graphs describing self-interaction due to dilaton $(a)$, two-form $(b)$ and linearized gravity $(c)$. The contributions from $a$ and $b$ are divergent and have different signs. The contribution of $c$ is zero.

\section{RENORMALIZATION}

The action of the proper fields upon the source string is described by the self-action terms in the Eq. (43) corresponding to the graphs shown on Fig. 2. the dilaton and the two-form lead to divergences while the contribution from the linearized gravity vanishes. Divergent terms can be absorbed by renormalization of the string tension parameter $\mu$ [43, 44]. We consider renormalization in the first order of the perturbation theory. Linearizing the string part of the action (2), one can split it into the sum:

$$
S=S_{s t}+S_{\phi}+S_{B}+S_{h}
$$

where $S_{s t}$ is Polyakov action with the bare tension parameter:

$$
S_{s t}=-\frac{\mu_{0}}{2} \int X_{a}^{\mu} X_{b}^{\nu} \eta_{\mu \nu} \eta^{a b} d^{2} \sigma
$$

and three other terms describe interaction with the dilaton, the two-form and the linearized gravity:

$$
\begin{aligned}
S_{\phi} & =-\mu_{0} \alpha \int \phi X_{a}^{\mu} X_{b}^{\nu} \eta_{\mu \nu} \eta^{a b} d^{2} \sigma \\
S_{B} & =-2 \pi f \int B_{\mu \nu} X_{c}^{\mu} X_{d}^{\nu} \epsilon^{c d} d^{2} \sigma \\
S_{h} & =-\frac{\mu_{0}}{2} \int X_{a}^{\mu} X_{b}^{\nu} \eta^{a b} h_{\mu \nu} d^{2} \sigma .
\end{aligned}
$$

Since we are working in the lowest order of the perturbation theory, the mapping functions $X^{\mu}$ here are quantities of zero order. In obtaining the last formula we used the following expansion:

$$
\gamma^{a b} \sqrt{-\gamma}=\eta^{a b}+h_{\mu \nu}\left(\frac{1}{2} X_{c}^{\mu} X^{\nu c} \eta^{a b}-X^{\mu a} X^{\nu b}\right)+\cdots
$$

Consider the first order dilaton field on the world-sheet of the source string

$$
\phi(\tau, \sigma)=\frac{\alpha \mu_{0}}{8 \pi^{2}} \int \frac{\mathrm{e}^{i q_{\mu}\left(d^{\mu}-X^{\mu}(\tau, \sigma)\right)} \delta(q u) \delta(q \Sigma)}{q^{2}} d^{4} q,
$$

where $q u=q_{\mu} u^{\mu}, q \Sigma=q_{\mu} \Sigma^{\mu}$ are the flat-space scalar products, and $q^{2}=q_{\mu} q^{\mu}$. Due to delta-functions, the scalar product in the exponential is constant:

$$
q_{\mu} X^{\mu}(\tau, \sigma)=(q d)+(q u) \tau+(q \Sigma) \sigma=(q d)=\text { const },
$$

so the integrand does not depend on $\sigma$ and $\tau$. The integral diverges as

$$
I=-\int \frac{\delta(q u) \delta(q \Sigma)}{q^{2}} d^{4} q=2 \pi \int_{0}^{\infty} \frac{d q_{\perp}}{q_{\perp}}
$$

where we used the frame (18) and introduced polar coordinates in the 2-plane orthogonal to $u^{\mu}$ and $\Sigma^{\mu}$ :

$$
q^{2}=(q u)^{2}-(q \Sigma)^{2}-q_{1}^{2}-q_{2}^{2}, \quad q_{1}^{2}+q_{2}^{2}=q_{\perp}^{2} .
$$

The integral logarithmically diverges at both ends. With the infrared (IR) an ultraviolet (UV) cut-off parameters $q_{\perp}^{\min }, q_{\perp}^{\max }$ one can write:

$$
I=2 \pi \ln \frac{q_{\perp}^{\max }}{q_{\perp}^{\min }} .
$$


Substituting this into the Eq. (59) and further into (55) we find the regularized dilaton part of the action:

$$
S_{\phi}^{r e g}=\frac{\mu_{0}^{2} \alpha^{2}}{4 \pi} \ln \frac{q_{\perp}^{\max }}{q_{\perp}^{\min }} \int X_{a}^{\mu} X_{b}^{\nu} \eta_{\mu \nu} \eta^{a b} d^{2} \sigma
$$

Since the functional is the same as the bare action functional $S_{s t}$, one can absorb divergencies by renormalization of $\mu_{0}$.

Similarly, the first order two-form field on the world-sheet of the source string reads:

$$
B^{\mu \nu}(\tau, \sigma)=\frac{f}{2 \pi} \int \frac{\mathrm{e}^{i q_{\mu}\left(d^{\mu}-X^{\mu}(\tau, \sigma)\right)} V^{\mu \nu} \delta(q u) \delta(q \Sigma)}{q^{2}} d^{4} q .
$$

This integral also diverges as (63). In view of the relation

$$
V^{\mu \nu} V_{\nu}^{\lambda}=-U^{\mu \nu}
$$

one can see that the action (56) also have the functional form of (54), namely

$$
S_{B}^{r e g}=-2 \pi f^{2} \ln \frac{q_{\perp}^{\max }}{q_{\perp}^{\min }} \int X_{a}^{\mu} X_{b}^{\nu} \eta_{\mu \nu} \eta^{a b} d^{2} \sigma
$$

Finally for the graviton $h_{\mu \nu}(\tau, \sigma)$ on the world-sheet we have the divergent integral

$$
h_{\mu \nu}(\tau, \sigma)=\frac{4 G \mu}{\pi} \int \frac{\mathrm{e}^{i q_{\mu}\left(d^{\mu}-X^{\mu}(\tau, \sigma)\right)} W_{\mu \nu} \delta(q u) \delta(q \Sigma)}{q^{2}} d^{4} q=-8 G \mu W_{\mu \nu} \ln \frac{q_{\perp}^{\max }}{q_{\perp}^{\min }},
$$

where $W_{\mu \nu}$ id given by the Eq. (35). However, substituting this into (57) one obtains zero in view of the identity

$$
W_{\mu \nu} U^{\mu \nu}=0 .
$$

Therefore, gravitational interaction of the strings does not lead to classical divergences in the lowest order of the perturbation theory. This result is conformal with previous results [41, 42] obtained with different tools.

Collecting the above formulas, we see that to remove self-interaction divergences one has merely to replace the tension parameter in the action (54) as follows:

$$
\left.\mu_{0}-\left(\frac{\mu_{0}^{2} \alpha^{2}}{2 \pi}-4 \pi f^{2}\right)\right) \ln \frac{q_{\perp}^{\max }}{q_{\perp}^{\min }}=\mu .
$$

Divergences due to the dilaton and the two-form have opposite signs. This reflects the fact that the scalar interaction is attractive, while interaction via the two-form is repulsive. If the Bogomolny-Prasad-Sommerfield (BPS) relation between the dilaton and the two-form caouplings is satisfied

$$
\frac{\mu^{2} \alpha^{2}}{2 \pi}=4 \pi f^{2}
$$

the divergent terms mutually cancel, and there is no renormalization at all, $\mu=\mu_{0}$ (for earlier work on this subject see refs. [41, 42, 43, 44, 45, 46]. Note that our dilaton coupling constant has dimension of length, the usual dimensionless constant $\bar{\alpha}$ (quantity of the order of unity) is related to it as

$$
\alpha^{2}=G \bar{\alpha}^{2} .
$$

It has to be noted that renormalization can be performed in a simple way only at the linearized level. When all non-linearities are taken into account, classical renormalizability of the bosonic string theory interacting with gravity is lost. In this paper we will be restricted by the second order calculation of radiation which involves only on-shell second order quantities. These are unaffected by the higher order renormalization effects. So, in what follows, we will use the value for the string tension renormalized in the first-order, and thus omit all self-interaction terms. 


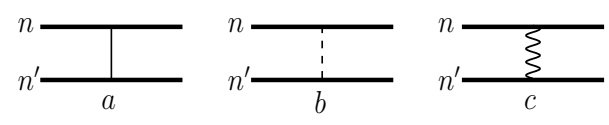

Рис. 3: Deformations of the strings world-sheets due to interactions via the dilaton (a), two-form (b) and linearized gravity (c); $n=1,2, n^{\prime}=1,2, n \neq n^{\prime}$.

\section{PERTURBATIONS OF THE STRING WORLD-SHEETS}

Now consider the first order perturbations of the mapping functions $X_{n}^{\mu}(\tau, \sigma), n=1,2$ caused by mutual interactions. We have to substitute into each string equation of motion (4) the first order fields generated by another string. It is convenient to use the following index convention: $n=1,2, n^{\prime}=1,2, n \neq n^{\prime}$. The total perturbation thus splits into three terms due to dilaton, two-from and graviton exchange:

$$
\stackrel{1}{X}_{n}^{\mu}=\stackrel{\phi}{X_{n}^{\mu}}+\stackrel{B}{X} \mu \stackrel{h}{X_{n}^{\mu}}
$$

as shown on Fig. 3. Let us start with dilaton exchange contribution (Fig. 3a). The corresponding world-sheet perturbation $\stackrel{1}{X}_{n}^{\mu}(\tau, \sigma)$ is the solution of the two-dimensional D'Alembert equation (following from the Eqs. (43/44)):

$$
\left(\partial_{\tau}^{2}-\partial_{\sigma}^{2}\right) \stackrel{\phi}{X}{ }_{n}^{\mu}=-2 \alpha\left[\left(\stackrel{\phi}{U^{\mu \nu}} \partial_{\nu}-\partial^{\mu}\right) \stackrel{1}{\phi}(x)\right]_{x=\stackrel{0}{X}_{n}(\tau, \sigma)},
$$

where the dilaton field generated by the string $n^{\prime}$ is taken on the world-sheet of the string $n$ :

$$
\left.\partial_{\mu} \stackrel{1}{\phi}\right|_{x=X_{n}(\tau, \sigma)} ^{0}=\frac{\alpha \mu}{8 \pi^{2} i} \int \frac{\mathrm{e}^{i q_{\lambda}\left(d_{n^{\prime}}^{\lambda}-X_{n}^{\lambda}(\tau, \sigma)\right)} \delta\left(q u_{n^{\prime}}\right) \delta\left(q \Sigma_{n^{\prime}}\right)}{q^{2}+2 i \epsilon q^{0}} q_{\mu} d^{4} q .
$$

Substituting (75) into (74) one can obtain the solution dividing the right hand side by the two-dimensional D'Alembert operator as follows:

$$
\stackrel{\phi}{X}_{n}^{\mu}(\tau, \sigma)=i \frac{\alpha^{2} \mu}{(2 \pi)^{2}} \int \frac{\Delta_{n^{\prime}}\left[q^{\mu}+\Sigma_{n}^{\mu}\left(q \Sigma_{n}\right)-u_{n}^{\mu}\left(q u_{n}\right)\right] \mathrm{e}^{-i q_{\lambda} \dot{X}_{n}^{\lambda}(\tau, \sigma)}}{q^{2}\left[\left(q u_{n}\right)^{2}-\left(q \Sigma_{n}\right)^{2}\right]} d^{4} q
$$

where

$$
\Delta_{n^{\prime}}=\mathrm{e}^{i q d_{n^{\prime}}} \delta\left(q u_{n^{\prime}}\right) \delta\left(q \Sigma_{n^{\prime}}\right)
$$

Note, that delta-functions in the integrand have support outside both the light cone $q^{2}=0$ and the surface $\left(q u_{n}\right)^{2}=$ $\left(q \Sigma_{n}\right)^{2}$ (except for the trivial point $q^{\mu}=0$ ), so the integral is finite.

Consider now the two-form interaction (Fig. 3b). We have to solve the equation

$$
\left(\partial_{\tau}^{2}-\partial_{\sigma}^{2}\right) \stackrel{B}{X_{n}^{\mu}}=\left.2 \pi f V_{n}^{\nu \lambda} \stackrel{1}{H}_{\nu \lambda}^{\mu}\right|_{x=X_{n}(\tau, \sigma)},
$$

where the three-form is

$$
\left.\stackrel{1}{H}_{\nu \lambda}^{\mu}\right|_{x=X_{n}^{0}(\tau, \sigma)}=\frac{f}{2 \pi i} \int \frac{\Delta_{n^{\prime}} q^{\{\mu} V_{n^{\prime}}^{\nu \lambda\}} e^{-i q_{\lambda} X_{n}^{\lambda}(\tau, \sigma)}}{q^{2}+2 i \epsilon q^{0}} d^{4} q,
$$

where curly brackets \{\} denote the cyclic permutation of indices. Again, dividing by the operator $\left(\partial_{\tau}^{2}-\partial_{\sigma}^{2}\right)$ one obtains:

$$
\stackrel{B}{X}_{n}^{\mu}(\tau, \sigma)=i \frac{f^{2}}{\mu} \int \frac{\Delta_{n^{\prime}} V_{n \nu \lambda} q^{\{\mu} V_{n^{\prime}}^{\nu \lambda\}} \mathrm{e}^{-i q_{\lambda} \stackrel{\mathrm{X}}{n}_{n}^{\lambda}(\tau, \sigma)}}{q^{2}\left[\left(q u_{n}\right)^{2}-\left(q \Sigma_{n}\right)^{2}\right]} d^{4} q .
$$

Similarly, the gravitational contribution is described by the equation:

$$
\left(\partial_{\tau}^{2}-\partial_{\sigma}^{2}\right) \stackrel{h}{X}{ }_{n}^{\mu}=\frac{\mu}{2} U_{n}^{\alpha \beta}\left[\partial^{\mu} \stackrel{1}{h} \alpha \beta-2 \partial_{\alpha} \stackrel{1}{h}_{\beta}^{\mu}-U_{n}^{\mu \nu}\left(\partial_{\nu} \stackrel{1}{h} \alpha \beta-2 \partial_{\beta} \stackrel{1}{h} \alpha \nu\right)\right]_{x=\stackrel{0}{X}_{n}(\tau, \sigma)},
$$


where the variation $\stackrel{1}{h}_{\mu \nu}$ is generated by the partner string $n^{\prime}$ :

$$
\left.\stackrel{1}{h}_{\mu \nu}\right|_{x=\stackrel{0}{X}_{n}(\tau, \sigma)}=\frac{4 \mu G}{\pi} \int \frac{\Delta_{n^{\prime}} W_{n^{\prime} \mu \nu} e^{-i q_{\lambda} \stackrel{0}{\lambda}_{n}^{\lambda}(\tau, \sigma)}}{q^{2}+2 i \epsilon q^{0}} d^{4} q .
$$

Solving this equation one finds:

$$
\stackrel{h}{X}_{n}^{\mu}(\tau, \sigma)=i \frac{2 \mu G}{\pi} \int \frac{\Delta_{n^{\prime}} U_{n \alpha \beta}\left[q^{\mu} W_{n^{\prime}}^{\alpha \beta}-2 W_{n^{\prime}}^{\mu \alpha} q^{\beta}-U_{n}^{\mu \nu}\left(q_{\nu} W_{n^{\prime}}^{\alpha \beta}-2 q^{\alpha} W_{n^{\prime} \nu}^{\beta}\right)\right] \mathrm{e}^{-i q_{\lambda} \stackrel{X}{n}_{n}^{\lambda}(\tau, \sigma)}}{q^{2}\left[\left(q u_{1}\right)^{2}-\left(q \Sigma_{1}\right)^{2}\right]} d^{4} q .
$$

It can be checked that the gauge condition (38) imposed in the beginning of the calculation holds indeed for each of the three separate contributions to the perturbed mapping functions.

\section{EFFECTIVE SOURCES OF RADIATION}

The first order fields $\stackrel{1}{\phi}, \stackrel{1}{B^{\mu \nu}}, \stackrel{1}{h}{ }^{\mu \nu}$ do not contain the radiative parts. Consider, e.g., the Fourrier-transform of the dilaton:

$$
\phi(k)=\int \phi(x) \mathrm{e}^{i k x} d^{4} x
$$

The retarded and advanced solutions of the first order wave equation (27) will read:

$$
\phi_{n}^{ \pm}(k)=2 \pi^{2} \mu \alpha \frac{\delta\left(k u_{n}\right) \delta\left(k \Sigma_{n}\right)}{k^{2} \pm 2 i \epsilon k^{0}} \mathrm{e}^{i k d_{n}} .
$$

The radiative part

$$
{\stackrel{1}{\phi_{n}^{\mathrm{rad}}}}^{\mathrm{r}}(k)=\frac{1}{2}\left(\stackrel{1}{\phi_{n}^{+}}(k)-\stackrel{1}{\phi}_{n}^{-}(k)\right)=-i \pi^{3} \mu \alpha \delta\left(k u_{n}\right) \delta\left(k \Sigma_{n}\right) \delta\left(k^{2}\right) \mathrm{e}^{i k d_{n}} .
$$

is the distribution having support only at the trivial point $k^{\mu}=0$ in the momentum space. Thus, to investigate radiation, we have to pass to the second order of the perturbation theory. The problem reduces to the construction of the source terms in the wave equations of the second order.

\section{A. Dilaton}

Consider the second order dilaton equation (47) in more details. The current at the right hand side contains the contributions localized on the string world-sheets (the upper line in (477) and the bulk contributions coming from the products of the first order dilaton, two-form and graviton fields (the lower line). The former contains the sum over the strings which can be understood as follows. One has to take the perturbations of the world-sheet mapping functions $X_{n}^{\mu}$ for each string $n=1,2$ due to first order field generated by the partner string $n=2,1$ respectively. These contributions are depicted by the graphs $a_{n}, b_{n}, c_{n}, n=1,2$ on the Fig. 4 . The external dilaton leg corresponds to the emitted dilaton with the momentum $k^{\mu}$ (in our classical treatment to the Fourrier-transform of the current), thus the terms in the sum in (47)) with $n=1$ are given by the graphs $a_{1}, b_{1}, c_{1}$ and those with $n=2-$ by the graphs $a_{2}, b_{2}, c_{2}$. Other upper line terms must be treated in a similar way: one has to take $U_{n}^{\mu \nu}$ for each $n=1,2$ and multiply by the graviton and dilaton perturbations caused by the other string $n=2,1$. These are depicted by the graphs $d_{n}, e_{n}, n=1,2$. In this way the self-action of the fields upon the string will be excluded.

On the contrary, the terms in the lower line of the Eq. (47) are non-local and not pairwise. Here the self-action terms also have to be excluded, so we take in the quadratic combinations only the products of the first order fields generated by different strings.

Consider first the contributions described by the graphs $a_{1}, b_{1}, c_{1}$ (dilaton emission from the first string line). The corresponding current reads:

$$
\stackrel{s t}{J}_{1}(x)=-\frac{\mu \alpha}{8 \pi} \int \stackrel{1}{X}_{1}^{\mu} \partial_{\mu} \delta^{4}\left[x-\stackrel{0}{X}_{1}(\tau, \sigma)\right] d^{2} \sigma,
$$




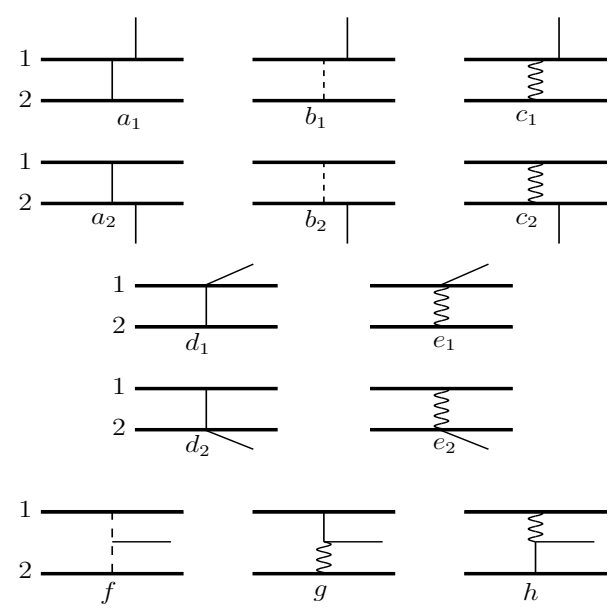

Рис. 4: The diagrams contributing to dilaton radiation in the second order of the perturbation theory: $a_{1}, b_{1}, c_{1}$ correspond to deformation of the first string and $a_{2}, b_{2}, c_{2}$ - to deformation of the second one; graphs $d, e$ stand for contact terms. Diagram $f$ corresponds to the product of (first order) two-form fields generated by the first and the second string, graphs $g, h-$ to mixed graviton-dilaton contributions.

where the perturbation of the mapping function of the first string ${ }_{X}^{\mu}$ is caused by the first order dilaton, two-form and graviton fields generated by the second string. Substituting the corresponding terms into (87) we obtain after some rearrangements:

$$
\stackrel{1}{X}_{1}^{\mu}(\tau, \sigma)=i \int \frac{Q_{1}^{\mu} \delta\left(q u_{2}\right) \delta\left(q \Sigma_{2}\right) \mathrm{e}^{i q\left(d_{2}-d_{1}-u_{1} \tau-\Sigma_{1} \sigma\right)}}{q^{2}\left[\left(q u_{1}\right)^{2}-\left(q \Sigma_{1}\right)^{2}\right]} d^{4} q .
$$

The vector $Q_{1}^{\mu}$ is the sum of three terms according to the decomposition described above:

$$
Q_{1}^{\mu}=\frac{\alpha^{2} \mu}{(2 \pi)^{2}} D_{1}^{\mu}+\frac{2 f^{2}}{\mu} Y_{1}^{\mu}+\frac{2 \mu G}{\pi} Z_{1}^{\mu}
$$

where the dilaton exchange contribution is

$$
D_{1}^{\mu}=q^{\mu}+\Sigma_{1}^{\mu}\left(q \Sigma_{1}\right)-u_{1}^{\mu}\left(q u_{1}\right)
$$

the two-form contribution is

$$
\begin{aligned}
Y_{1}^{\mu} & =q^{\mu}\left[\left(u_{1} u_{2}\right)\left(\Sigma_{1} \Sigma_{2}\right)-\left(\Sigma_{1} u_{2}\right)\left(u_{1} \Sigma_{2}\right)\right]+\Sigma_{2}^{\mu}\left[\left(q u_{1}\right)\left(u_{2} \Sigma_{1}\right)-\left(\Sigma_{1} q\right)\left(u_{1} u_{2}\right)\right]+ \\
& +u_{2}^{\mu}\left[\left(u_{1} \Sigma_{2}\right)\left(\Sigma_{1} q\right)-\left(q u_{1}\right)\left(\Sigma_{1} \Sigma_{2}\right)\right],
\end{aligned}
$$

and the graviton contribution is

$$
\begin{aligned}
Z_{1}^{\mu}= & q^{\mu}\left[\left(u_{1} u_{2}\right)^{2}+\left(\Sigma_{1} \Sigma_{2}\right)^{2}-\left(u_{1} \Sigma_{2}\right)^{2}-\left(u_{2} \Sigma_{1}\right)^{2}-2\right]+\left[u_{1}^{\mu}\left(q u_{1}\right)-\Sigma_{1}^{\mu}\left(q \Sigma_{1}\right)\right] \cdot \\
& {\left[\left(u_{1} u_{2}\right)^{2}-\left(\Sigma_{1} \Sigma_{2}\right)^{2}-\left(u_{1} \Sigma_{2}\right)^{2}+\left(u_{2} \Sigma_{1}\right)^{2}+2\right]-2\left[u_{1}^{\mu}\left(q \Sigma_{1}\right)+\Sigma_{1}^{\mu}\left(q u_{1}\right)\right]\left[\left(u_{1} u_{2}\right)\left(u_{2} \Sigma_{1}\right)-\left(\Sigma_{1} \Sigma_{2}\right)\left(u_{1} \Sigma_{2}\right)\right]-} \\
- & 2 u_{2}^{\mu}\left[\left(u_{1} u_{2}\right)\left(q u_{1}\right)-\left(\Sigma_{1} u_{2}\right)\left(q \Sigma_{1}\right)\right]+2 \Sigma_{2}^{\mu}\left[\left(u_{1} \Sigma_{2}\right)\left(q u_{1}\right)-\left(\Sigma_{1} \Sigma_{2}\right)\left(q \Sigma_{1}\right)\right] .
\end{aligned}
$$

Consider the Fourier-transform

$$
J(k)=\int J(x) \mathrm{e}^{i k x} d^{4} x
$$

with $k^{2}=0$ (on-shell condition for the massless particle). Substituting the above expressions and integrating over the world-sheet of the first string one obtains two more delta-functions:

$$
\int \mathrm{e}^{i(k-q)\left(u_{1} \tau-\Sigma_{1} \sigma\right)} d \tau d \sigma=(2 \pi)^{2} \delta\left[(k-q) u_{1}\right] \delta\left[(k-q) \Sigma_{1}\right],
$$


so totally we will have the product of four delta-functions in the integrand:

$$
\Lambda_{1}(q, k)=\delta\left(q u_{1}\right) \delta\left(q \Sigma_{1}\right) \delta\left[(k-q) u_{2}\right] \delta\left[(k-q) \Sigma_{2}\right] .
$$

Now consider the contribution $J_{2}(k)$ coming from the second string (the graphs $a_{2}, b_{2}, c_{2}$ on Fig.4). Obviously it can be obtained from the previous result by interchanging indices $1 \leftrightarrow 2$ elsewhere. In this case we will get the product of the $\delta$-functions in the form

$$
\Lambda_{2}(q, k)=\delta\left(q u_{2}\right) \delta\left(q \Sigma_{2}\right) \delta\left[(k-q) u_{1}\right] \delta\left[(k-q) \Sigma_{1}\right]
$$

It is convenient to cast this second integral into the same form as the previous one. For this it is sufficient to shift the integration variable in $J_{2}(k)$ as follows: $q^{\mu} \rightarrow k^{\mu}-q^{\mu}$. Since $\Lambda_{1}(q, k)=\Lambda_{2}(k-q, k)$, we will get the same product of the $\delta$-functions, so finally we can present the total contribution of the first six graphs as follows

$$
\stackrel{s t}{J}(k)=\stackrel{s t}{J}_{1}(k)+\stackrel{s t}{J}_{2}(k)=\int \Pi(q, k)\left(\frac{\stackrel{s t}{\Theta}_{1}(q)}{q^{2}}+\frac{\stackrel{s t}{\Theta}_{2}(k-q)}{(k-q)^{2}}\right) d^{4} q,
$$

where

$$
\Pi(q, k)=\mathrm{e}^{i\left(d_{1} k+q\left(d_{2}-d_{1}\right)\right)} \Lambda_{1}(q, k),
$$

and

$$
\stackrel{s t}{\Theta}_{1}(q)=\frac{\alpha}{\left[\left(q \Sigma_{1}\right)^{2}-\left(q u_{1}\right)^{2}\right]}\left[\frac{\alpha^{2} \mu^{2}}{8 \pi}\left(k D_{1}\right)+\pi f^{2}\left(k Y_{1}\right)+G \mu^{2}\left(k Z_{1}\right)\right] \text {. }
$$

To get the function $\stackrel{s t}{\Theta}_{2}$ from $\stackrel{s t}{\Theta}_{1}$ one merely has to interchange the indices 1,2 labelling vectors in the scalar products changing simultaneously $q^{\mu} \rightarrow(k-q)^{\mu}$.

The contributions of the next four graphs $d_{1}, d_{2}, e_{1}, e_{2}$ are computed similarly. The resulting "contact" term in the source can be presented again in the form (97) with equal contributions from two strings

$$
\stackrel{c t}{\Theta}_{1}(q)=\stackrel{c t}{\Theta}_{2}(q)=\frac{\alpha^{3} \mu^{2}}{8 \pi}+G \mu^{2} \alpha\left[\left(u_{1} u_{2}\right)^{2}+\left(\Sigma_{1} \Sigma_{2}\right)^{2}-\left(u_{1} \Sigma_{2}\right)^{2}-\left(u_{2} \Sigma_{1}\right)^{2}-2\right] .
$$

Now consider the bulk terms (the second line in (47)) which are due to non-linear field interactions dilaton-two-form and dilaton-graviton. Their contribution is illustrated by the graphs $f$ and $e_{n}$, the second being pairwise. In quantum theory terms they can be interpreted as the coalescence of two virtual axions into the on-shell dilaton, and the coalescence of the off-shell dilaton and graviton into the on-shell dilaton. We have to compute the Fourrier-transform of the bulk current

$$
\stackrel{b}{J}(x)=\frac{\alpha}{24 \pi} \stackrel{1}{H}_{\mu \nu \lambda} \stackrel{1}{H}^{\mu \nu \lambda}+\frac{1}{4 \pi} \partial_{\mu}\left[\left(\stackrel{1}{h}^{\mu \nu}-\frac{1}{2} \eta^{\mu \nu} \stackrel{1}{h}\right) \partial_{\nu} \stackrel{1}{\phi}\right] .
$$

Here in the products $\stackrel{1}{H}_{\mu \nu \lambda} \stackrel{1}{H}^{\mu \nu \lambda}$ and $\stackrel{1}{\phi}_{h}^{h}$, we have to substitute one field generated by the first string and another by the second one.

In spite of the different different appearance of the bulk terms as compared to the world-sheet terms, one can cast them into the unique form (97) as well. Consider the corresponding transformations for the first term in (101) depicted by the graph $f$ on Fig. 4. The first order quantities to be substituted here read:

$$
\stackrel{1}{H}_{n}^{\mu \nu \lambda}(x)=\frac{f}{2 i \pi} \int \frac{\Delta_{n} q^{\{\mu} V_{n}^{\nu \lambda\}} \mathrm{e}^{-i q x}}{q^{2}} d^{4} q
$$

(We can omit the $\epsilon$-term in the denominator indicating the position of the pole since the resulting integral does not depend on its shift from the real axis). Now let us calculate the contraction

$$
\begin{aligned}
\int \mathrm{e}^{i k x} \stackrel{1}{H}_{\mu \nu \lambda} \stackrel{1}{H}^{\mu \nu \lambda} d^{4} x & =-(2 \pi f)^{2} \int \frac{\Delta_{1}(q) \Delta_{2}\left(q^{\prime}\right) q_{\{\mu} V_{1 \nu \lambda\}} q^{\prime \mu \mu} V_{2}^{\nu \lambda\}} \mathrm{e}^{i\left(k-q-q^{\prime}\right) x}}{q^{2} q^{\prime 2}} d^{4} q= \\
& =-(2 \pi f)^{2} \int \frac{\Delta_{1}(q) \Delta_{2}(k-q) q_{\{\mu} V_{1 \nu \lambda\}}(k-q)^{\{\mu} V_{2}{ }^{\nu \lambda\}} \mathrm{e}^{i\left(k-q-q^{\prime}\right) x}}{q^{2}(k-q)^{2}} d^{4} q
\end{aligned}
$$




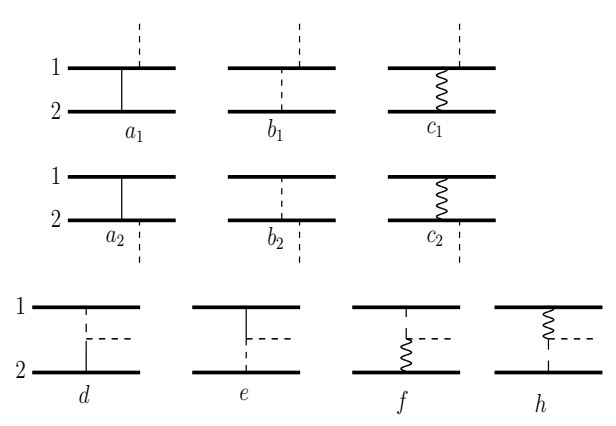

Рис. 5: The second order amplitudes of the two-form emission. The graphs $a, b, c$ show contributions from deformations of the string world-sheets, the graphs $d, e, f, h$ - the quadratic bulk terms.

We are interested in the on-shell value of $k^{\mu}$, i.e. $k^{2}=0$. In this case one can write

$$
\frac{1}{q^{2}(k-q)^{2}}=\left(\frac{1}{(q-k)^{2}}-\frac{1}{q^{2}}\right) \frac{1}{2 k q}
$$

Using this relation one can cast the above contraction into the form (97) with convention that the argument $q^{\mu}$ is used for the $n=1$ terms, and $(k-q)^{\mu}$ for the $n=2$ terms.

Performing similar calculations for the graphs $g_{1}, g_{2}$ and combining with the above, we obtain the total bulk term in the form (97) with $\Theta=\stackrel{b}{\Theta}$ :

$$
\stackrel{b}{\Theta}_{1}=\pi f^{2} \alpha\left[\frac{\left(Y_{1} k\right)}{(k q)}-\left(u_{1} u_{2}\right)\left(\Sigma_{1} \Sigma_{2}\right)+\left(\Sigma_{1} u_{2}\right)\left(u_{1} \Sigma_{2}\right)\right]+\mu^{2} \alpha G \frac{\left(k u_{2}\right)^{2}-\left(k \Sigma_{2}\right)^{2}+\left(k u_{1}\right)^{2}-\left(k \Sigma_{1}\right)^{2}}{(k q)},
$$

with the rule of getting $\stackrel{b}{\Theta}_{2}$ from $\stackrel{b}{\Theta}_{1}$ the same as before. In obtaining this expression we have used the delta-functions in the integrand fixing $\left(q u_{1}\right)=\left(q \Sigma_{1}\right)=0$ and $\left(q u_{2}\right)=\left(k u_{2}\right),\left(q \Sigma_{1}\right)=\left(k \Sigma_{1}\right)$.

\section{B. Two-form}

The source term in the equation (8) for the second order two-form field can be presented as the sum of ten graphs shown on Fig.5. Here we have string contributions corresponding to exchange by the dilaton, two-form and graviton, the bulk terms describing coalescence of virtual quanta to the on-shell axion, but no contact term because of absence of multi-leg vertices associated with two-form. The string terms $a_{n}, b_{n}, c_{n}$ are computed as the Fourrier-transform of the currents

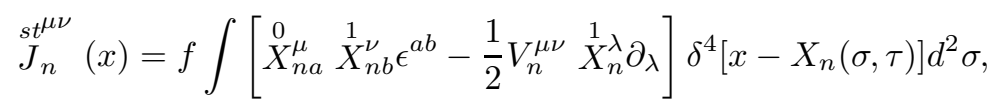

where perturbations of the mapping functions $X_{n}^{\mu}, n=1,2$ are generated by the partner string $n=2,1$ respectively. Using the same rearrangements as before, we obtain

$$
{ }^{s t} J^{\mu \nu}(k)=\stackrel{s t}{J}_{1}^{\mu \nu}(k)+\stackrel{s t}{J}_{2}^{\mu \nu}(k)=\int \Pi(q, k)\left(\frac{\stackrel{s t}{\Theta}_{1}^{\mu \nu}(q)}{q^{2}}+\frac{\stackrel{s t}{\Theta}_{2}^{\mu \nu}(k-q)}{(k-q)^{2}}\right) d^{4} q,
$$

where

$$
\begin{aligned}
\Theta_{1}^{s \nu}(q)= & -\frac{1}{\left[\left(q \Sigma_{1}\right)^{2}-\left(q u_{1}\right)^{2}\right]}\left[8 \pi^{2} \frac{f^{3}}{\mu}\left(\left(q \Sigma_{1}\right) u_{1}^{[\mu} Y_{1}^{\nu]}-\left(q u_{1}\right) \Sigma_{1}^{[\mu} Y_{1}^{\nu]}-\frac{1}{2} V_{1}^{\mu \nu}\left(Y_{1} k\right)\right)+\right. \\
& +\frac{1}{2} \alpha^{2} f \mu\left(\left(q \Sigma_{1}\right) u_{1}^{[\mu} D_{1}^{\nu]}-\left(q u_{1}\right) \Sigma_{1}^{[\mu} D_{1}^{\nu]}-\frac{1}{2} V_{1}^{\mu \nu}\left(D_{1} k\right)\right)+ \\
& +8 \pi f \mu G\left(\left(q \Sigma_{1}\right) u_{1}^{[\mu} Z_{1}^{\nu]}+\left(q u_{1}\right) \Sigma_{1}^{[\mu}\left(Z_{1}^{\nu]}-\frac{1}{2} V_{1}^{\mu \nu}\left(Z_{1} k\right)\right)\right],
\end{aligned}
$$




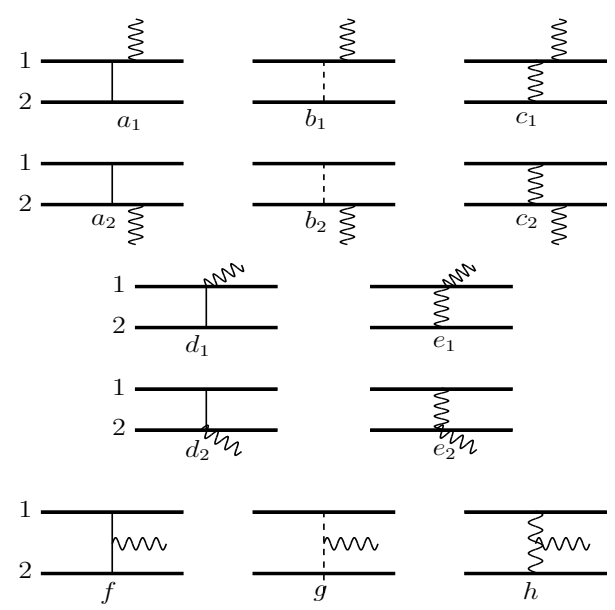

Pис. 6: The second order amplitudes of the graviton emission. The sum of all graphs is zero on the graviton mass-shell.

and to get the second string term we must interchange indices $1 \leftrightarrow 2$ and momenta $q^{\mu} \leftrightarrow k^{\mu}-q^{\mu}$.

The bulk terms $d_{n}, e_{n}$ are the Fourrier-transform of the bulk current

$$
\stackrel{f}{J}(x)_{\mu \nu}=\frac{1}{4 \pi} \partial_{\lambda}\left[\stackrel{1}{H}^{\lambda}{ }_{\mu \nu}\left(4 \alpha \stackrel{1}{\phi}-\frac{1}{2} \stackrel{1}{h}\right)\right],
$$

where again we have to take the products of fields generated by different strings. The result can be cast into the form (107) with

$$
\begin{aligned}
\stackrel{b}{\Theta}_{1}^{\mu \nu} & \left.=-\frac{8 \pi f \mu G}{(k q)}\left[V_{1}^{\mu \nu}(k q)+\left(q^{[\mu} u_{1}^{\nu]}\right)\left(\Sigma_{1} q\right)-\left(q^{[\mu} \Sigma_{1}^{\nu]}\right)\left(u_{1} q\right)-V_{2}^{\mu \nu}(k q)+\left(q^{[\mu} u_{2}^{\nu]}\right)\left(\Sigma_{2} k\right)-\left(q^{[\mu} \Sigma_{2}^{\nu]}\right)\left(u_{2} k\right)\right)\right]- \\
& \left.-\frac{f \alpha^{2} \mu}{2(k q)}\left[V_{1}^{\mu \nu}(k q)\right)-\left(q^{[\mu} u_{1}^{\nu]}\right)\left(\Sigma_{1} q\right)+\left(q^{[\mu} \Sigma_{1}^{\nu]}\right)\left(u_{1} q\right)-V_{2}^{\mu \nu}(k q)-\left(q^{[\mu} u_{2}^{\nu]}\right)\left(\Sigma_{2} k\right)+\left(q^{[\mu}\right) \Sigma_{2}^{\nu]}\left(u_{2} k\right)\right],
\end{aligned}
$$

and the same rule for the second term.

\section{Graviton}

The source (52) in the second order graviton equation can be treated along the same line. It includes contributions of the thirteen graphs shown on Fig. 6. It turns out, however, that the projection of the total on-shell $\left(k^{2}=0\right)$ current onto the graviton transverse polarization states gives zero. The reason lies in the dimensionality of the space transversal to the string: the configuration of the parallel strings is reduced to that of point particles in the $1+2$ theory, where there is no on-shell transverse gravitational degrees of freedom. As it was shown in [37], the transformation to the parallel string configuration is always possible for a superluminal moving intersecting strings. So there is no gravitational radiation in this case and we do not give here the details of the calculation.

\section{RADIATION}

Total radiation four-momentum loss can be presented in a standard way through the on-shell Fourrier transform of the source current, the vector $k^{\mu}=(\omega, \mathbf{k}), \omega=|\mathbf{k}|$ playing the role of the radiation four-momentum. In the case of the dilaton one obtains the following explicitly Lorentz-covariant expression:

$$
P_{(\phi)}^{\mu}=\frac{16}{\pi} \int k^{\mu} \frac{k^{0}}{\left|k^{0}\right|}\left|J_{(\phi)}(k)\right|^{2} \delta\left(k^{2}\right) d^{4} k,
$$

and similarly for the two-form

$$
P_{(B)}^{\mu}=\frac{1}{\pi} \int k^{\mu} \frac{k^{0}}{\left|k^{0}\right|}\left|J_{\alpha \beta}(k)\right|^{2} \delta\left(k^{2}\right) d^{4} k
$$


Alternatively, the latter quantity can be presented as a square of the polarization projection of the current. Indeed, in three space dimensions the two-form field propagating along the wave vector $\mathbf{k}$ has a unique polarization state

$$
e_{i j}=\frac{1}{\sqrt{2}}\left(e_{i}^{\theta} e_{j}^{\varphi}-e_{i}^{\varphi} e_{j}^{\theta}\right), \quad i, j=1,2,3,
$$

where $\mathbf{e}^{\theta}$ and $\mathbf{e}^{\varphi}$ are two unit vectors orthogonal to $\mathbf{k}$ and to each other:

$$
\mathbf{e}^{\varphi} \cdot \mathbf{e}^{\theta}=0, \quad \mathbf{k} \cdot \mathbf{e}^{\varphi}=\mathbf{k} \cdot \mathbf{e}^{\theta}=0 .
$$

Using antisymmetry and transversality of the two-form current $k^{\mu} J_{\mu \nu}(k)=0$, and the completeness condition

$$
e_{i}^{\theta} e_{j}^{\theta}+e_{i}^{\varphi} e_{j}^{\varphi}=\delta_{i j}-k_{i} k_{j} / \omega^{2}
$$

one finds

$$
P_{(B)}^{\mu}=\frac{1}{\pi} \int k^{\mu} \frac{k^{0}}{\left|k^{0}\right|}\left|J_{(B)}^{i j}(k) e_{i j}\right|^{2} \delta\left(k^{2}\right) d^{4} k .
$$

Integrating over $k^{0}$, we finally obtain

$$
\begin{aligned}
P_{(\phi)}^{\mu} & =\frac{16}{\pi} \int \frac{k^{\mu}}{|\mathbf{k}|}\left|J_{(\phi)}(\mathbf{k})\right|^{2} d^{3} k, \\
P_{(B)}^{\mu} & =\frac{1}{\pi} \int \frac{k^{\mu}}{|\mathbf{k}|}\left|J_{(B)}(\mathbf{k})\right|^{2} d^{3} k,
\end{aligned}
$$

where

$$
J_{(B)}(\mathbf{k})=J_{(B)}^{i j}(k) e_{i j}
$$

with $k^{0}=|\mathbf{k}|$. In what follows we shall use the parametrization of three-vectors by the spherical angles: $\mathbf{k}=$ $\omega[\sin \theta \cos \varphi, \sin \theta \sin \varphi, \cos \theta], \mathbf{e}^{\varphi}=[-\sin \varphi, \cos \varphi, 0], \mathbf{e}^{\theta}=[\cos \theta \cos \varphi, \cos \theta \sin \varphi,-\sin \theta]$.

\section{A. Cerenkov condition}

With our conventions, the radiation amplitudes associated with the first string contain the integral

$$
I_{1}=\int \frac{\delta\left(q u_{2}\right) \delta\left(q \Sigma_{2}\right) \delta\left[(k-q) u_{1}\right] \delta\left[(k-q) \Sigma_{1}\right] f(q) \mathrm{e}^{i q d}}{q^{2}} d^{4} q
$$

where $d^{\mu}=d_{2}^{\mu}-d_{1}^{\mu}$, and $f(q)$ - is some regular function of $q$. In the Lorentz frame where $u_{1}^{\mu}=(1,0,0,0), \Sigma_{1}^{\mu}=$ $(0,0,0,1), u_{2}^{\mu}=\gamma(1,0,-v \cos \alpha, v \sin \alpha), \Sigma_{2}^{\mu}=(0,0, \sin \alpha, \cos \alpha)$, one can integrate over $q^{0}$ using

$$
\delta\left[(k-q) u_{1}\right]=\delta\left(q^{0}-\omega\right) .
$$

Two other $\delta$-functions

$$
\begin{aligned}
& \delta\left(q u_{2}\right)=\gamma^{-1} \delta\left(\omega+q_{y} v \cos \alpha-q_{z} v \sin \alpha\right), \\
& \delta\left(q \Sigma_{2}\right)=\gamma^{-1} \delta\left(q_{y} \sin \alpha+q_{z} v \cos \alpha\right),
\end{aligned}
$$

can be used to fix the values of $q_{y}, q_{z}$ :

$$
q_{y}=-\frac{\omega \cos \alpha}{v}, \quad q_{z}=\frac{\omega \sin \alpha}{v} .
$$

The remaining $\delta$-function

$$
\delta\left[(k-q) \Sigma_{1}\right]=\delta\left(k_{z}-q_{z}\right)=\delta\left(k_{z}-\frac{\omega \sin \alpha}{v}\right)
$$


no more depends on $q$, but rather restricts the value of the wave vector of radiation $k_{z}$ :

$$
k_{z}=\frac{\omega \sin \alpha}{v}=v_{p}
$$

This is Cerenkov's condition for an emitted massless wave. Indeed, in our frame an effective source of radiation is moving along z-axis with the velocity $v_{p}$, thus the quantity

$$
\cos \theta=\frac{k_{z}}{\omega}=\frac{1}{v_{p}}
$$

defines Cerenkov's angle of emission if $v_{p}>1$, i.e. the source is super-luminal. Thus, radiation arises if the string relative velocity $v$ and the inclination angle $\alpha$ satisfy the Cerenkov condition

$$
\sin \alpha<v .
$$

Given $v$, this condition will always be satisfied for sufficiently small $\alpha$. In particular, it is identically fulfilled for $\alpha=0$, i.e. for parallel strings. Moreover, it can be shown that, if $v_{p}>1$, the Lorentz frame and the world sheet coordinates $\tau_{n}, \sigma_{n}$ always exist such that two string look parallel [37]. In the frame where the first string is at rest and streched along the $z$-axis, an effective source moves in $z$-direction, and radiation will be emitted along the Cerenkov cone around the $z$-axis with an angle

$$
\theta_{\mathrm{Ch}}=\arccos \frac{v}{\sin \alpha} .
$$

The remaining integral over $q_{x}$ in (120) can be evaluated using contour integration. With our choice of coordinates, the scalar product $q^{\mu} d_{\mu}=-q_{x} d$, so we have the integral over $q_{x}$

$$
I_{1}=\int \frac{\mathrm{e}^{-i q_{x} d} f\left(q_{x}\right) d q_{x}}{q_{x}^{2}+p^{2}}=\frac{\pi}{p} f(-i p) \mathrm{e}^{-\kappa d}
$$

where

$$
p=\sqrt{q_{y}^{2}+q_{z}^{2}-q_{0}^{2}}=\frac{\omega}{\gamma v} .
$$

For the second term in (97) containing the pole $(k-q)^{-2}$ one obtains the same fixed values of $q^{0}, q_{y}, q_{z}$, but the integration over $q_{x}$ gives the value

$$
q_{x}=k_{x}+\frac{\omega \xi}{i v}, \quad \xi=\cos \alpha+v \sin \theta \sin \phi .
$$

Summarizing the above results, we obtain:

$$
\int\left(\frac{\Theta_{1}(q)}{q^{2}}+\frac{\Theta_{2}(k-q)}{(k-q)^{2}}\right) \mathrm{e}^{i k d_{1}+i q d_{2}} d^{4} q=\left[E_{1} \Theta_{1}\left(q_{1}\right)+(\gamma \xi)^{-2} E_{2} \Theta_{2}\left(k-q_{2}\right)\right] \delta\left(\cos \theta-\cos \theta_{\mathrm{Ch}}\right),
$$

where

$$
E_{1}=\mathrm{e}^{i k d_{1}-\omega d /(v \gamma)}, \quad E_{2}=\mathrm{e}^{i k d_{2}-\omega d \xi / v},
$$

and the following complex vectors are introduced:

$$
\begin{aligned}
q_{1}{ }^{\mu} & =\frac{\omega}{v}[v,-i / \gamma,-\cos \alpha, \sin \alpha], \\
k^{\mu}-q_{2}^{\mu} & =\frac{\omega \xi}{v}[0, i, 1,0] .
\end{aligned}
$$

The presence of the delta-function at the right hand side of (133) means that the total radiation loss is infinite. This could be expected since we deal with the stationary motion of an infinitely long strings. So it is natural to consider the radiation loss per unit length of the string at rest. Redefining the currents as

$$
J(\mathbf{k})=I(\mathbf{k}) \delta\left(\cos \theta-\cos \theta_{\mathrm{Ch}}\right),
$$


and using the identity

$$
\delta^{2}\left(\cos \theta-\cos \theta_{\mathrm{Ch}}\right)=\frac{L \omega}{2 \pi} \delta\left(\cos \theta-\cos \theta_{\mathrm{Ch}}\right),
$$

where $L$ is the normalization length, we find

$$
\begin{aligned}
& \mathcal{P}_{(\phi)}^{\mu}=L^{-1} P_{(\phi)}^{\mu}=\frac{8}{\pi^{2}} \int k^{\mu}\left|I_{(\phi)}(\mathbf{k})\right|^{2} \delta\left(\cos \theta-\cos \theta_{\mathrm{Ch}}\right) d^{3} k \\
& \mathcal{P}_{(B)}^{\mu}=L^{-1} P_{(B)}^{\mu}=\frac{1}{2 \pi^{2}} \int k^{\mu}\left|I_{(B)}(\mathbf{k})\right|^{2} \delta\left(\cos \theta-\cos \theta_{\mathrm{Ch}}\right) d^{3} k
\end{aligned}
$$

\section{B. Relativistic peaking and spectrum enhancement}

According to (133), there is a frequency cut-off due to exponential factors (134). Actually the amplitude is superposition of two terms which can be associated with contributions of two strings (this can not be taken literally: in the second order of the perturbation theory the superposition principle does not hold). Two terms exhibit different frequency cut-off. The first sting term has a cut-off

$$
\omega \lesssim \frac{v \gamma}{d}
$$

which does not depend on the radiation angle, while the second one exhibits a $\varphi$-dependent cut-off:

$$
\omega \lesssim \frac{v}{d \xi}=\frac{v}{d(\cos \alpha+v \sin \theta \sin \varphi)}
$$

This means that the angular distribution of radiation on the Cerenkov cone is anisotropic. This feature becomes especially pronounced in the ultrarelativistic case.

In view of the identity

$$
\cos ^{2} \alpha=v^{2} \sin ^{2} \theta+\gamma^{-2}
$$

which holds on the radiation cone, in the ultrarelativistic limit $\gamma \rightarrow \infty$ the quantity $\xi$ has a sharp minimum at $\varphi=-\pi / 2$ corresponding to the direction of the moving string in the rest frame of the first string:

$$
\left.\xi \approx \frac{1}{2 \gamma^{2} \cos \alpha}\left(1+\beta^{2} \gamma^{2} \cos \alpha^{2}\right)\right],
$$

where $\beta=\pi / 2+\varphi \ll 1$. Due to the factor $\xi^{-2}$ in the second term in (119) the Cerenkov radiation is peaked around the direction $\varphi=-\pi / 2$ within the narrow angular region

$$
\beta \lesssim \gamma^{-1}
$$

Moreover, the frequency range associated with the second terms is substantially larger in the ultrarelativistic limit than that associated with the first term. Indeed, if $\kappa=\gamma \cos \alpha \gg 1$, one has:

$$
\xi \approx \frac{1}{2 \gamma \kappa}\left(1+\kappa^{2} \beta^{2}\right)
$$

so the frequency range extends up to the frequency

$$
\omega \lesssim \frac{\gamma \kappa}{d}
$$

in the angular region (145). Therefore, radiation exhibits relativistic peaking in the forward direction in the same way as radiation of the ultrarelativistic particle. This could be expected, since relativistic peaking has purely kinematical nature. 


\section{Dilaton radiation}

Collecting the world-sheet contributions to radiation amplitudes we obtain after integration over $q$ :

$$
\begin{aligned}
\stackrel{s t}{I}(k)+\stackrel{c t}{I}(k)=\frac{\alpha}{\omega^{2}}\left\{\frac{\alpha^{2} \mu^{2}}{8}\left(E_{1}\left[1-\frac{i \cos \phi+\kappa \sin \phi}{\gamma v \sin \theta}\right]+\frac{E_{2}}{\gamma^{2} \xi^{2}}\left[\frac{i \mathrm{e}^{-i \varphi}}{\gamma v \sin \theta}+2 \xi \gamma\right]\right)+\right. \\
+\pi^{2} f^{2}\left(E_{1} \frac{\sin \phi+i \kappa \cos \varphi}{\gamma v \sin \theta}-\frac{E_{2}}{\gamma^{2} \xi^{2}} \frac{\gamma v \sin \theta+i \kappa \mathrm{e}^{-i \varphi}}{\gamma v \sin \theta}\right)+ \\
\left.+\mu^{2} G \pi\left(E_{1}[(\kappa \sin \varphi-i \cos \phi)+\gamma v \sin \theta]+\frac{E_{2}}{\gamma^{2} \xi^{2}} i e^{i \varphi}\right) \gamma v \sin \theta\right\}
\end{aligned}
$$

Here the upper line corresponds to the dilaton exchange, the second line - to the two-form and the last line to the graviton exchange. The bulk contribution after integration reads:

$$
\begin{aligned}
\stackrel{b}{I}(k) & =\frac{\pi^{2} f^{2} \alpha}{\gamma \xi}\left[E_{1}(\gamma \kappa \xi-1-i \gamma v \sin \theta \cos \varphi)+i E_{2} \gamma v \sin \theta \mathrm{e}^{i \phi}\right]+ \\
& +\frac{G \mu^{2} \pi}{\gamma \xi}\left[E_{1}(\gamma v \sin \theta+\kappa \sin \phi-i \cos \phi)+E_{2} i \mathrm{e}^{i \phi}\right] 2 \gamma \kappa v \sin \theta .
\end{aligned}
$$

Consider first the Cerenkov's threshold $v=\sin \alpha$, when the radiation cone shrinks to $\theta_{\mathrm{Ch}}=0$. This corresponds to $\kappa=1, \xi=1 / \gamma$. Since in this limit $k d=0$, the exponents become equal $E_{1}=E_{2}$ and the bulk term vanishes. The graviton contribution to (148) vanishes too, while the dilaton and two-form contributions differs only by the coefficients. The total radiation amplitude at the threshold will be given by

$$
I_{\mathrm{thr}}(k)=\frac{\alpha}{8 \omega^{2}} E_{1}\left(3 \alpha^{2} \mu^{2}-8 \pi^{2} f^{2}\right) .
$$

Integrating the expression (139) for $\mu=0$ (the energy loss rate) over the angles in $d^{3} k=\omega^{2} d \omega d \cos \theta d \varphi$, we obtain the infrared-divergent integral over frequencies. Introducing the inverse correlation distance $\Delta$ as an infrared cutoff parameter one finds:

$$
\mathcal{P}_{\mathrm{thr}}=\frac{\alpha^{2}}{4 \pi}\left(3 \alpha^{2} \mu^{2}-8 \pi^{2} f^{2}\right)^{2} \int_{\Delta^{-1}}^{\infty} \frac{d \omega}{\omega} \exp \left(-2 \frac{\omega d}{v \gamma}\right)
$$

In the BPS limit (71) one has

$$
\mathcal{P}_{\mathrm{thr}}=\frac{\mu^{4} \alpha^{6}}{\pi} \int_{\Delta^{-1}}^{\infty} \frac{d \omega}{\omega} \exp \left(-2 \frac{\omega d}{v \gamma}\right)
$$

Integrating this over frequencies we obtain the total radiation rate in terms of the integral exponential function (see Appendix):

$$
\mathcal{P}_{\mathrm{thr}}=\frac{\mu^{4} \alpha^{6}}{\pi} \operatorname{Ei}\left(1, \frac{2 d}{v \gamma \Delta}\right) .
$$

For small impact parameters $d \ll v \gamma \Delta$ this expression can be approximated by the logarithm

$$
\mathcal{P} \approx \frac{\mu^{4} \alpha^{6}}{\pi} \ln \left(\frac{v \gamma \Delta}{2 d \mathrm{e}^{C}}\right)
$$

where $C$ is the Euler constant, $\mathrm{e}^{C}=1.781072418$. For large impact parameters, $d \gg v \gamma \Delta$, radiation exponentially falls off:

$$
\operatorname{Ei}\left(1, \frac{2 d}{v \gamma \Delta}\right) \approx \frac{2 d}{v \gamma \Delta} \exp \left(-\frac{2 d}{v \gamma \Delta}\right)
$$

For $\theta \neq 0$ the radiation amplitudes are more complicated. Note, that in our reference frame the first string is at rest while the second one is moving. For this reason the radiation amplitudes are not symmetric with respect to two strings. As it was observed in the previous section, in the ultrarelativistic case the frequency range associated with 
the factor $E_{2}$ is much larger than that that with $E_{1}$, and in this limit the $E_{2}$ terms are dominant. Collecting the dominant terms we obtain for the total amplitude of the dilaton emission in the ultrarelativistic case

$$
\begin{array}{r}
I(\mathbf{k})=\frac{\alpha E_{2}}{\gamma^{2} \xi^{2} \omega^{2}}\left\{\frac{\alpha^{2} \mu^{2}}{8}\left(\frac{i \mathrm{e}^{-i \phi}}{\gamma v \sin \theta}+2 \gamma \xi\right)-\pi^{2} f^{2}(1+\right. \\
\left.+\frac{i \cos \alpha \mathrm{e}^{-i \phi}}{v \sin \theta}-i \xi \gamma^{2} v \sin \theta \mathrm{e}^{i \phi}\right)+ \\
\left.+\mu^{2} G \pi i \gamma v \sin \theta(1+2 \kappa \gamma \xi) \mathrm{e}^{i \phi}\right\} .
\end{array}
$$

Substituting this into (139) and taking into account the angular peaking near $\phi=-\pi / 2$ we obtain the spectral-angular distribution of radiation per unit length in the vicinity of this direction:

$$
\frac{d \mathcal{P}}{d \omega d \beta}=\frac{32 \alpha^{2} \kappa^{2}}{\omega} \frac{\left(\Omega_{1}+\Omega_{2} \kappa^{2} \beta^{2}\right)^{2}}{\left(1+\kappa^{2} \beta^{2}\right)^{4}} \exp \left(-\frac{\omega d\left(1+\kappa^{2} \beta^{2}\right)}{\gamma \kappa}\right),
$$

where

$$
\Omega_{1}=4 G \mu^{2} \kappa^{2}+\pi f^{2} \kappa \quad \Omega_{2}=2 G \mu^{2} \kappa^{2}+\pi f^{2} \kappa+\frac{\alpha^{2} \mu^{2}}{4 \pi} .
$$

Dividing by $\omega$ we will get also the number of emitted dilatons

$$
\frac{d \mathcal{N}}{d \omega d \beta}=\frac{1}{\omega} \frac{d \mathcal{P}}{d \omega d \beta}
$$

Note, that the graviton, axion and dilaton exchange terms entering into the above expressions for the parameters $\Omega_{1,2}$ exhibit different behavior in the invariant Lorentz factor $\kappa$, the dominant as $\kappa \rightarrow \infty$ being the gravitational term.

The spectrum exhibits an infrared divergence, and in the forward direction $\beta=0$ it extends up to $\omega \sim \omega$ max, where

$$
\omega_{\max }=\frac{\gamma \kappa}{d}
$$

Integrating over frequencies with the infrared cut-off $\Delta^{-1}$ we obtain the angular distribution of radiation:

$$
\frac{d \mathcal{P}}{d \beta}=32 \alpha^{2} \kappa^{2} \frac{\left(\Omega_{1}+\Omega_{2} \kappa^{2} \beta^{2}\right)^{2}}{\left(1+\kappa^{2} \beta^{2}\right)^{4}} \operatorname{Ei}\left(1, \frac{d\left(1+\beta^{2} \gamma \kappa\right)}{\gamma \kappa \Delta}\right) .
$$

Since the integral exponential function decays exponentially for large argument, the total radiation is peaked around $\beta=0$ within the angle

$$
\beta \lesssim \sqrt{\gamma \kappa}
$$

One can also obtain the spectral distribution of radiation extending the integration domain over $\beta$ in (158) to the full axis in view of the exponential decay of the integrand:

$$
\frac{d \mathcal{P}}{d \omega}=\omega \frac{d \mathcal{N}}{d \omega} \frac{2 \pi \alpha^{2} d}{3 \gamma}\left[\Omega_{1}^{2} F_{1}(z)+2 \Omega_{1} \Omega_{2} F_{2}(z)+\Omega_{2}^{2} F_{3}(z)\right]
$$

where $z=\frac{\omega d}{\gamma \kappa}$ and the functions $F_{i}$ are expressed in terms of the probability integral (226):

$$
\begin{aligned}
& F_{1}(z)=\left(8 z^{2}-16 z+30\right) \frac{\mathrm{e}^{-z}}{\sqrt{\pi z}}-\left(8 z^{2}-12 z+18-\frac{15}{z}\right) \operatorname{erfc}(\sqrt{z}) \\
& F_{2}(z)=\left(-8 z^{2}-8 z+6\right) \frac{\mathrm{e}^{-z}}{\sqrt{\pi z}}+\left(8 z^{2}+12 z-6+\frac{3}{z}\right) \operatorname{erfc}(\sqrt{z}) \\
& F_{3}(z)=\left(8 z^{2}+32 z+6\right) \frac{\mathrm{e}^{-z}}{\sqrt{\pi z}}-\left(8 z^{2}+36 z+18-\frac{3}{z}\right) \operatorname{erfc}(\sqrt{z}) .
\end{aligned}
$$

For small frequencies $\omega \ll \gamma \kappa / d$ these functions grows as

$$
F_{1}(z) \sim \frac{15}{z}, \quad F_{2}(z) \sim \frac{3}{z}, \quad F_{3}(z) \sim \frac{3}{z},
$$


while for large $z$ they exponentially decay:

$$
F_{1}(z) \sim \frac{48 \mathrm{e}^{-z}}{\sqrt{\pi z^{3}}}, \quad F_{2}(z) \sim-\frac{57 \mathrm{e}^{-z}}{2 \sqrt{\pi z^{5}}}, \quad F_{3}(z) \sim \frac{105 \mathrm{e}^{-z}}{2 \sqrt{\pi z^{5}}} .
$$

To regularize the infrared divergence one has to introduce the cut-off length $\Delta$. After integration over frequencies $\omega$ from $\Delta^{-1}$ to infinity we obtain the total dilaton radiation rate in the ultrarelativistic limit:

$$
\mathcal{P}=\frac{2 \pi \alpha^{2} \kappa}{3}\left[\left(\Omega_{1}^{2} f_{1}(y)+2 \Omega_{1} \Omega_{2} f_{2}(y)+\Omega_{2}^{2} f_{3}(y)\right)\right],
$$

where three new functions are introduced

$$
\begin{aligned}
& \left.f_{1}(y)=5 f(y)+\operatorname{erfc}(\sqrt{y})\right)\left(\frac{8}{3} y^{3}-6 y^{2}+18 y+\frac{37}{2}\right)-\frac{\mathrm{e}^{-y} \sqrt{y}}{\pi}\left(\frac{8}{3} y^{2}-\frac{22}{3} y+23\right), \\
& f_{2}(y)=f(y)-\operatorname{erfc}(\sqrt{y})\left(\frac{8}{3} y^{3}+6 y^{2}-6 y-\frac{5}{2}\right)+\frac{\mathrm{e}^{-y} \sqrt{y}}{\pi}\left(\frac{8}{3} y^{2}+\frac{14}{3} y-7\right), \\
& f_{3}(y)=f(y)+\operatorname{erfc}(\sqrt{y})\left(\frac{8}{3} y^{3}+18 y^{2}+18 y+\frac{1}{2}\right)-\frac{\mathrm{e}^{-y} \sqrt{y}}{\pi}\left(\frac{8}{3} y^{2}+\frac{50}{3} y+11\right),
\end{aligned}
$$

where

$$
y=\frac{d}{\gamma \kappa \Delta},
$$

and the function $f(y)$ is expressed through the generalized hypergeometric function (230)

$$
f(y)=12 \sqrt{\frac{y}{\pi}}{ }_{2} F_{2}\left(\frac{1}{2}, \frac{1}{2} ; \frac{3}{2}, \frac{3}{2} ;-y\right)-3 \ln \left(4 y \mathrm{e}^{C}\right) .
$$

For small $y$ these functions grow as

$$
f_{1}(y) \sim 15 \ln \frac{1}{y}, \quad f_{2,3}(y) \sim 3 \ln \frac{1}{y},
$$

while for large $y$ they tend to zero in view of the asymptotic relation (233).

Similar expressions can be obtained for the total number of dilatons:

$$
\mathcal{N}=\frac{2 \pi \alpha^{2} d}{3 \gamma}\left(\Omega_{1}^{2} \mathcal{F}_{1}(y)+2 \Omega_{1} \Omega_{2} \mathcal{F}_{2}(y)+\Omega_{2}^{2} \mathcal{F}_{3}(y)\right)
$$

where three more functions are introduced

$$
\begin{aligned}
& \mathcal{F}_{1}(y)=\operatorname{erfc}(\sqrt{y})\left(4 y^{2}-12 y-39+\frac{15}{y}\right)-\frac{\mathrm{e}^{-y}}{\pi \sqrt{y}}\left(4 y^{2}-14 y-30\right)-6 f(y), \\
& \mathcal{F}_{2}(y)=\operatorname{erfc}(\sqrt{y})\left(-4 y^{2}-12 y-9+\frac{3}{y}\right)+\frac{\mathrm{e}^{-y}}{\pi \sqrt{y}}\left(4 y^{2}+10 y+6\right)-2 f(y), \\
& \mathcal{F}_{3}(y)=\operatorname{erfc}(\sqrt{y})\left(4 y^{2}+36 y+9+\frac{3}{y}\right)-\frac{\mathrm{e}^{-y}}{\pi \sqrt{y}}\left(4 y^{2}+34 y-6\right)-6 f(y),
\end{aligned}
$$

tending to zero at infinity, and

$$
\mathcal{F}_{1} \sim \frac{15}{y}, \quad \mathcal{F}_{2,3} \sim \frac{3}{y}
$$

for small $y$.

As we have noted, in the ultrarelativistic limit the dominant contribution to radiation amplitude comes from the graviton exchange term. Leaving only this contribution we find for the radiation rate:

$$
\mathcal{P}^{(\phi)}=\frac{8}{3} \pi G^{2} \alpha^{2} \mu^{4} \kappa^{5} g(y),
$$




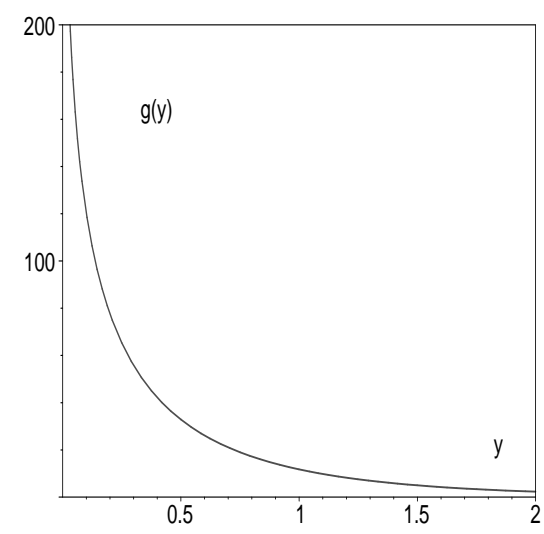

Рис. 7: g(y).

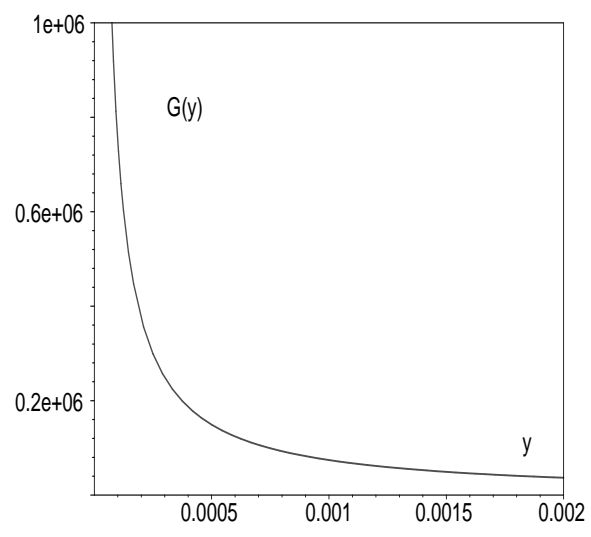

Рис. 8: $G(y)$.

where

$$
g(y)=25 f(y)+\operatorname{erfc}(\sqrt{y})\left(\frac{8}{3} y^{3}-30 y^{2}+114 y+\frac{169}{2}\right)-\frac{\mathrm{e}^{-y} \sqrt{y}}{\pi}\left(\frac{8}{3} y^{2}-\frac{94}{3} y+131\right) .
$$

Similary, for the dilaton number:

$$
\begin{aligned}
& \mathcal{N}^{(\phi)}=\frac{8 d}{3 \gamma} \pi G^{2} \alpha^{2} \mu^{4} \kappa^{4} \mathcal{G}(y) \\
& \left.\mathcal{G}_{(y)}=\operatorname{erfc}(\sqrt{y})\right)\left(+4 y^{2}-60 y-183+\frac{75}{y}\right)-\frac{\mathrm{e}^{-y}}{\pi \sqrt{y}}\left(4 y^{2}-62 y-150\right)-38 f(y)
\end{aligned}
$$

The corresponding numerical curves are shown on the Figs 78 . For small $y$ one has:

$$
\begin{aligned}
\mathcal{P}^{(\phi)} & =200 \pi G^{3} \bar{\alpha}^{2} \mu^{4} \kappa^{5} \ln \left(\frac{\gamma \kappa \Delta}{d}\right), \\
\mathcal{N}^{(\phi)} & =200 \pi G^{3} \bar{\alpha}^{2} \mu^{4} \kappa^{5} \Delta,
\end{aligned}
$$

where we introduced the dimensionless dilaton coupling constant. These quantities rapidly grow with increasing Lorentz factor of the collision $\gamma$ (recall that $\kappa=\gamma \cos \alpha$ where $\alpha$ is the angle between the strings). Thus, Cerenkov radiation is greatly enhanced for ultrarelativistic velocities.

\section{Two-form radiation}

After integration over the momentum $q$, the string contribution to the two-form radiation amplitude (107) takes the form:

$$
\begin{array}{r}
\text { st } \\
I(k)=\frac{4 \sqrt{2} \pi^{3} f^{3}}{\mu \gamma v \sin \theta}\left(E_{1}(\cos \varphi-i \kappa \sin \varphi)+\frac{E_{2}}{\gamma^{2} \xi^{2}}\left[\left(i v \xi \gamma^{2} \sin \theta-\mathrm{e}^{-i \varphi}\right)\right)+\right. \\
+\frac{\alpha^{2} f \mu \pi \sqrt{2}}{2 \gamma v \sin \theta}\left(E_{1}(i \sin \varphi-\kappa \cos \varphi)+\frac{E_{2}}{\gamma^{2} \xi^{2}}\left(\kappa \mathrm{e}^{-i \varphi}-i v \gamma \sin \theta-2 \gamma^{3} \xi v^{2} \sin ^{2} \theta \cos \varphi\right)\right)+ \\
+4 \sqrt{2} \pi^{2} f \mu G \gamma v \sin \theta\left(E_{1}(\kappa \cos \varphi+i \sin \varphi)-\frac{E_{2}}{\gamma^{2} \xi^{2}}\left[i \gamma v \sin \theta+\kappa \mathrm{e}^{i \varphi}-2 \cos \varphi \gamma \xi\right]\right) .
\end{array}
$$

The bulk amplitude (107) will read:

$$
\stackrel{b}{I}(k)=\left(\frac{\alpha^{2} f \mu \pi \sqrt{2}}{2}+4 \sqrt{2} \pi^{2} f \mu G \gamma v \sin \theta\right)\left(\frac{E_{1}}{\gamma \xi}[(1-2 \gamma \xi) \gamma v \sin \theta \cos \varphi-i]+\frac{E_{2}}{\xi}(2 v \sin \theta \cos \varphi-i \xi)\right) .
$$


Consider first the Cerenkov threshold $v=\sin \alpha$. In the above expressions the graviton exchange term in the string term vanishes, and the bulk term is zero. The remaining string amplitudes due to the dilaton and the two-form exchange simplify as follows:

$$
I_{\mathrm{thr}}(k)=\frac{i \pi f}{\sqrt{2} \mu \omega^{2}} E_{1}\left(8 \pi^{2} f^{2}-3 \alpha^{2} \mu^{2}\right) .
$$

Substituting this into (140) and integrating over the angles we obtain the spectral distribution of the two-form radiation on the Cerenkov threshold:

$$
\mathcal{P}_{\text {thr }}=\frac{\pi f^{2}}{2 \mu^{2}}\left(8 \pi^{2} f^{2}-3 \alpha^{2} \mu^{2}\right)^{2} \int_{\Delta^{-1}}^{\infty} \frac{d \omega}{\omega} \exp \left(-2 \frac{\omega d}{v \gamma}\right),
$$

where $\Delta$ the infrared cutoff frequency. After integration over frequencies one finds:

$$
\mathcal{P}_{\text {thr }}=\frac{4(2 \pi)^{5} f^{6}}{\mu^{2}} \operatorname{Ei}\left(1, \frac{2 d}{v \gamma \Delta}\right) .
$$

For small impact parameters $d \ll v \gamma \Delta$ this expression can be approximated by

$$
\mathcal{P}_{\mathrm{thr}} \approx \frac{4(2 \pi)^{5} f^{6}}{\mu^{2}} \ln \left(\frac{v \gamma \Delta}{2 d \mathrm{e}^{C}}\right)
$$

for $d \gg v \gamma \Delta$ one can use

$$
\operatorname{Ei}\left(1, \frac{2 d}{v \gamma \Delta}\right) \approx \frac{2 d}{v \gamma \Delta} \exp \left(-\frac{2 d}{v \gamma \Delta}\right)
$$

Now consider the case of the arbitrary Cerenkov angles in the ultrarelativistic limit. Leaving only the relativistic string contribution, we obtain:

$$
\begin{array}{r}
I(\mathbf{k})=\frac{f E_{2}}{\gamma^{2} \xi^{2} \omega^{2}}\left\{\frac{4 \sqrt{2} \pi^{3} f^{2}}{\mu \gamma v \sin \theta}\left(i \gamma^{2} \xi v \sin \theta-i \mathrm{e}^{-i \phi}\right)+\frac{\pi \mu \alpha^{2}}{\sqrt{2}}\left(\frac{\kappa \mathrm{e}^{-i \phi}+\gamma v \sin \theta}{\gamma v \sin \theta}-i \gamma^{2} \xi^{2}\right)-\right. \\
\left.\left.-4 \sqrt{2} \pi^{2} G \mu\left[i\left(1-\kappa^{2}\right)+\kappa \gamma v \sin \theta e^{i \phi}\right)-i \gamma^{2} \xi^{2}\right]\right\} .
\end{array}
$$

Substituting this into (140) we obtain the spectral-angular distribution in the vicinity of $\phi=\pi / 2$

$$
\frac{d \mathcal{P}}{d \omega d \beta}=\omega \frac{d \mathcal{N}}{d \omega d \beta}=\frac{64 \pi^{4} \kappa^{2} f^{2}}{\omega} \frac{\chi_{B}^{2}\left(1-\kappa^{2} \beta^{2}\right)^{2}+4 \kappa^{4} \beta^{2}\left(\chi_{h} \kappa^{2}-\chi_{D}\right)^{2}}{\left(1+\kappa^{2} \beta^{2}\right)^{4}} \exp \left(-\frac{\omega d\left(1+\kappa^{2} \beta^{2}\right)}{\gamma \kappa}\right),
$$

where

$$
\chi_{D}=\frac{\mu \alpha^{2}}{8 \pi^{2}}, \quad \chi_{B}=\frac{f^{2}}{\mu}, \quad \chi_{h}=\frac{G \mu}{\pi} .
$$

Integrating over the angles we get:

$$
\begin{aligned}
\frac{d \mathcal{P}}{d \omega} & =\frac{16 \pi^{5} d}{3 \gamma}\left[F_{4}(z) \chi_{B}^{2}+F_{2}(z)\left(\chi_{h} \kappa^{2}-\chi_{D}\right)^{2}\right] \\
\frac{d \mathcal{N}}{d \omega} & =\frac{16 \pi^{5} d^{2} f^{2}}{3 \gamma^{2} \kappa z}\left[F_{4}(z) \chi_{B}^{2}+F_{2}(z)\left(\chi_{h} \kappa^{2}-\chi_{D}\right)^{2}\right]
\end{aligned}
$$

where

$$
F_{4}=\left(8 z^{2}+8 z+6\right) \frac{\mathrm{e}^{-z}}{\sqrt{\pi z}}-\left(8 z^{2}+12 z+6-3 / z\right) \operatorname{erfc}(\sqrt{z}),
$$

and $F_{2}$ is given by (165). For small and large frequencies one has:

$$
F_{4}(z) \sim \frac{3}{z}, \quad F_{4}(z) \sim \frac{12 \mathrm{e}^{-z}}{\sqrt{\pi z^{3}}}
$$


respectively. Finally, integrating over frequencies we find the total two-form radiation rate and the number of axions:

$$
\begin{aligned}
\mathcal{P}^{(B)} & =\frac{16 \pi^{5} \kappa f^{2}}{3}\left(\chi_{B}^{2} f_{4}(y)+\left(\chi_{h} \kappa^{2}-\chi_{D}\right)^{2} f_{2}(y)\right), \\
\mathcal{N}^{(B)} & =\frac{16 \pi^{5} d f^{2}}{3 \gamma}\left(\chi_{B}^{2} \mathcal{F}_{4}(y)+\left(\chi_{h} \kappa^{2}-\chi_{D}\right)^{2} \mathcal{F}_{2}(y)\right),
\end{aligned}
$$

where

$$
\begin{aligned}
& f_{4}(y)=f(y)+\operatorname{erfc}(\sqrt{y})\left(\frac{8}{3} y^{3}+6 y^{2}+5 y+\frac{7}{2}\right)-\frac{\mathrm{e}^{-y} \sqrt{y}}{\pi}\left(\frac{8}{3} y^{2}+\frac{14}{3} y+5\right) \\
& \mathcal{F}_{4}(y)=\operatorname{erfc}(\sqrt{y})\left(4 y^{2}+12 y-3+\frac{3}{y}\right)-\frac{\mathrm{e}^{-y}}{\pi \sqrt{y}}\left(4 y^{2}+10 y-6\right)-2 f(y) .
\end{aligned}
$$

For small $y$

$$
\mathcal{F}_{4} \sim \frac{3}{y}, \quad f_{4}(y) \sim 3 \ln \frac{1}{y}
$$

while for large $y$ both functions tend to zero.

The result of the calculation of the axion Cerenkov radiation in the flat space-time [40] is reproduced putting $\chi_{D}=\chi_{h}=0$. It reads

$$
\mathcal{P}_{0}^{(B)}=\frac{16 \pi^{5} \kappa f^{6}}{3 \mu^{2}} f_{4}(y) .
$$

In our case the dominant contribution comes form the graviton exchange. In the BPS limit one has

$$
\chi_{D}=\chi_{B}, \quad \chi_{h}=\frac{8}{\bar{\alpha}^{2}} \chi_{B},
$$

where $\bar{\alpha}$ is the dimensionless dilaton coupling constant. For large $\kappa$ the leading term is

$$
\mathcal{P}^{(B)}=\frac{2^{10} \pi^{5} \kappa^{5} f^{6}}{3 \bar{\alpha}^{4} \mu^{2}} f_{2}(y) .
$$

In the most interesting case of small $y f_{2} \simeq f_{4}$, so the the ratio

$$
\frac{\mathcal{P}^{(B)}}{\mathcal{P}_{0}^{(B)}}=\frac{64 \kappa^{4}}{\bar{\alpha}^{4}}
$$

can be large, e.g. for $\bar{\alpha}=1$ and $\kappa=5$ it is equal to $4 \cdot 10^{7}$.

The dominant term for the number of axions is

$$
\mathcal{N}=\frac{2^{10} \pi^{5} \kappa^{4} f^{6} d}{3 \gamma \bar{\alpha}^{4} \mu^{2}} \mathcal{F}_{2}(y) .
$$

The numerical curves $f_{2}(y), \mathcal{F}_{2}(y)$ are shown on Figs. 9, 10, For small $y$ one has:

$$
\begin{aligned}
\mathcal{P}^{(B)} & =\frac{2^{10} \pi^{5} \kappa^{5} f^{6}}{\bar{\alpha}^{4} \mu^{2}} \ln \left(\frac{\gamma \kappa \Delta}{d}\right) \\
\mathcal{N} & =\frac{2^{10} \pi^{5} \kappa^{5} f^{6} \Delta}{\bar{\alpha}^{4} \mu^{2}}
\end{aligned}
$$

\section{COSMOLOGICAL ESTIMATES}

Cosmic strings are formed as a network of long strings of the size comparable to the horizon size. Colliding strings intercommute and form closed loop. At some stage the scale-invariant string network is formed consisting of long strings and loops which move freely with relativistic velocities. Evolution of cosmic superstring networks was recently 


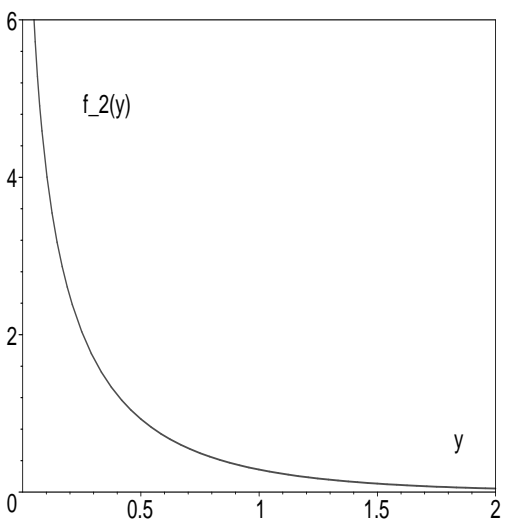

Рис. 9: $f_{2}(y)$.

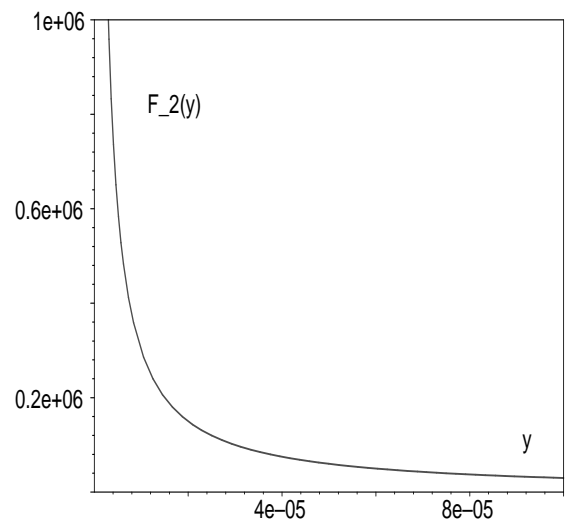

Рис. 10: $\mathcal{F}_{2}(y)$.

discussed in [20]-[24]. In many respects cosmic superstrings are similar to the gauge theory cosmic strings, with some distinctions, however. In particular, for gauge theory strings the probability of the formation of loops $P$ is of the order of unity, whereas for F-strings $P \sim 10^{-3}$ and for and D-strings $P \sim 10^{-1}$. The cosmic superstring network has a scaling solution and the characteristic scale is proportional to the square root of the reconnection probability. A typical separation between two long strings is comparable to the horizon size $t$ (we use the standard cosmological units), $\zeta(t) \simeq \sqrt{P} t$. The results of numerical simulation show that the network of long strings reaches an energy density

$$
\rho_{s}=\frac{\mu}{\sqrt{P} t^{2}} .
$$

Let us estimate the energy loss of long strings due to Cerenkov radiation of dilatons and axions. Consider an ensemble of randomly oriented straight strings moving chaotically in space. Let choose one target string between them and introduce the Lorentz frame where it is at rest. Other strings will have different orientations and velocities, and we can characterize them very roughly as moving in three orthogonal directions with equal probability. Since the dependence of the Cerenkov radiation on the inclination angle $\alpha$ is smooth, we can use for an estimate the particular result obtained for parallel strings $(\alpha=0)$ introducing an effective fraction $\nu$ of "almost" parallel strings taking into account the effect of the angular spread. Assuming $N$ to be the number of strings in the normalization volume $V=L^{3}$, we have to integrate the radiation energy released $\mathcal{P}$ in the collision with the impact parameter $d=x$ over the plane perpendicular to the target string with the measure $N / L^{2} \cdot 2 \pi x d x$. To estimate the radiation power per unit time we then have to divide the integrand by the impact parameter. Multiplying this quantity by the total number of strings $N$ to get the radiation energy released per unit time within the normalization volume, we obtain for the Cerenkov luminosity:

$$
Q_{C}=\int_{0}^{L} \mathcal{P} \nu \frac{N}{L^{2}} \frac{N}{V} 2 \pi d x
$$

For BPS strings we use as $\mathcal{P}$ the leading relativistic terms (180) and (207). Taking into account that the string number density is related to the energy density (212) via

$$
\frac{N}{V}=\frac{\rho_{s}}{\mu L}
$$

and assuming for a rough estimate $L \sim \Delta \sim t$, where $t$ is cosmological time, we obtain

$$
\begin{aligned}
& Q_{C}^{(\phi)} \simeq \frac{16}{3} \pi^{2} G^{3} \bar{\alpha}^{2} \mu^{4} \gamma^{7} \nu S_{1}(w) \frac{1}{P t^{3}}, \\
& Q_{C}^{(B)} \simeq \frac{2^{11} \pi^{6} \gamma^{7} f^{6} \nu}{3 \bar{\alpha}^{4} \mu^{2}} S_{2}(w) \frac{1}{P t^{3}},
\end{aligned}
$$

where

$$
S_{1}(w)=\int_{0}^{w} g(y) d y, \quad S_{2}(w)=\int_{0}^{w} f_{2}(y) d y, \quad w=\frac{L}{\gamma^{2} \Delta} .
$$




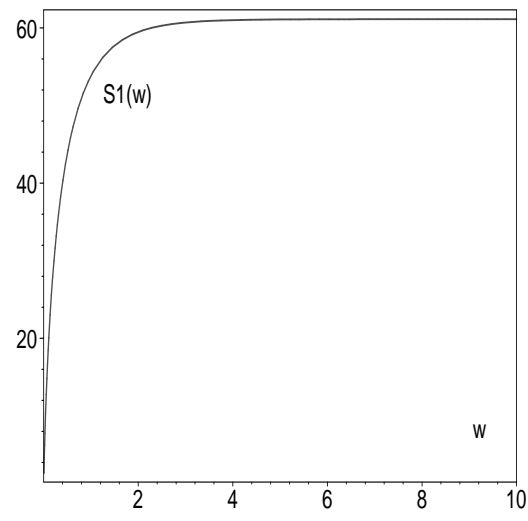

Рис. 11: $S_{1}(w), w=L /\left(\gamma^{2} \Delta\right)$

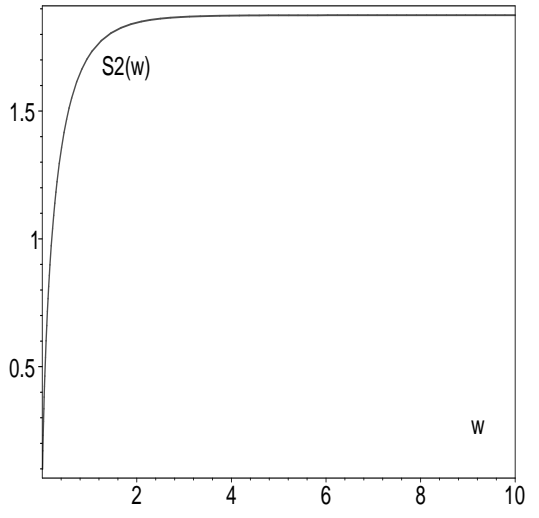

Рис. 12: $S_{2}(w)$

The exact values of these integrals are given in the Appendix and shown on Figs 1112. Note that the realistic value of $\gamma$ is of the order of unity, so applying our formulas obtained in the limit $\gamma \gg 1$ is only an order of magnitude estimate.

Now we can calculate the energy density $\varepsilon_{C}$ of Cerenkov radiation as a function of time in the radiation dominated Universe. The energy density of massless fields scales with the Hubble parameter $H$ as $H^{-4}$, so we have to solve the equation

$$
\frac{d \varepsilon_{C}}{d t}=-4 H \varepsilon_{C}+Q_{C},
$$

where $H=\frac{1}{2 t}$. Thus we obtain for the energy density of the Cerenkov dilaton and two-form radiation:

$$
\begin{aligned}
& \varepsilon_{C}^{(\phi)} \simeq \frac{16}{3} \pi^{2} G^{3} \bar{\alpha}^{2} \mu^{4} \gamma^{7} \nu S_{1}(w) \frac{\ln \left(t / t_{0}\right)}{P t^{2}}, \\
& \varepsilon_{C}^{(B)} \simeq \frac{2^{11} \pi^{6} \gamma^{7} f^{6} \nu}{3 \bar{\alpha}^{4} \mu^{2}} S_{2}(w) \frac{\ln \left(t / t_{0}\right)}{P t^{2}},
\end{aligned}
$$

where $t_{0}$ - the initial time of the long string formation. Finally, using the approximate formulas (236)237) valid in the relativistic case, we find:

$$
\begin{aligned}
& \varepsilon_{C}^{(\phi)} \simeq 800 \pi^{2} G^{3} \bar{\alpha}^{2} \mu^{4} \gamma^{5} \ln \gamma \nu \frac{\ln \left(t / t_{0}\right)}{P t^{2}} \\
& \varepsilon_{C}^{(B)} \simeq \frac{2^{12} \pi^{6} f^{6} \gamma^{5} \ln \gamma \nu}{\bar{\alpha}^{4} \mu^{2}} \frac{\ln \left(t / t_{0}\right)}{P t^{2}} .
\end{aligned}
$$

Comparing the last expression for axions with the flat space result [40] we observe an enhancement due to higher power of the Lorentz factor and bigger numerical coefficient. This difference is due to the fact that here the main contribution to the first order interaction between the strings comes from gravitational force which is proportional to energy. In view of the previous analysis [40] we can conclude that Cerenkov radiation from long strings is non-negligible effect in the cosmic superstring network. More detailed analysis will be given elsewhere.

\section{CONCLUSION}

In this paper we have studied in detail Cerenkov radiation from moving straight strings interacting with dilaton, two-form and gravity. Formation of the faster-than-light sources in the system of randomly oriented moving straight strings is rather generic. As we have shown, these sources have a collective nature and arise due to deformations of the strings world-sheets caused by their interactions via massless fields. These deformations propagate with superluminal velocities if the inclination angle is sufficiently small, for parallel strings the source velocity is infinite. Radiation wave vectors lie on the Cerenkov cone in the same way as in the case of Cerenkov radiation of point charges in dielectric media. 
One interesting feature related to dimensionality of a string compared to a point charge is the absence of gravitational radiation in four space-time dimensions. This is related to the fact that the space transverse to the straight string is twodimensional, so the emitted massless fields must live in $1+2$ dimensional space-time rather than in four-dimensional. As it is well-known, gravity in 1+2 dimensions does not contain free gravitons, this is why one can expect gravitational radiation from straight strings to vanish. In higher dimensions this objection does not work, so Cerenkov gravitational radiation can be expected in space-time dimensions higher than four. In four dimensions Cerenkov mechanism works for the dilaton and the two-form field which is equivalent to a pseudoscalar.

To avoid complications due to possibility of "physical" string intersections (leading to the well studied processes of intercommutation and formation of loops) we consider the "collision" of strings moving in parallel planes. At each instant of time there exists a point of minimal separation between the strings, and it is this point which may propagate with the superluminal velocity. When interaction between the strings via dilaton, two-form and gravity is taken into account, strings get deformed in the vicinity of this point, these deformations contribute to an effective radiation source. Another contribution comes from tensions associated with the first order fields which give rise to these deformations. The string deformations give contributions localized on the world-sheets, while the field stresses give bulk contributions. Both have the same order of magnitude.

Cerenkov radiation from strings has some peculiar features in the highly relativistic region. We have shown that in this case radiation exhibits strong beaming on the Cherenkov cone in the direction of the fast string in the rest frame of the target string. The main radiation frequency is proportional to the inverse impact parameter, but in the ultrarelativistic case the spectrum is enhanced to high frequencies proportional to the square of the Lorentz-factor of the collision. It is shown that in this limit gravitational interaction between string dominates and gives the main contribution to the effective sources of dilaton and two-form radiation.

Cerenkov's mechanism can be regarded as an analog of the bremsstrahlung of point charges in electrodynamics, which gives the main contribution to radiation in plasma. In the string case, however, there is another radiation mechanism due to existence of the internal string dynamics: radiation from oscillating loops. This effect is of the first order in couplings between the string and massless fields. Cerenkov radiation arises only in the second order in these coupling, so presumably it is less important. But as we have shown here, it has stronger dependence on the Lorentz factor of the string collision, so it must become dominant for highly relativistic strings. Also, in the cosmic string network it is a pairwise effect which gives contribution to the radiation loss proportional to the square of the density of strings. Our rough cosmological estimates indicate that Cerenkov radiation is a non-negligible effect in the cosmic string context indeed.

\section{Acknowledgments}

This work was supported by RFBR grant 02-04-16949.

\section{APPENDIX}

Here we collect properties of some special functions used in the main text.

\section{A. Integral exponential function}

The definition:

$$
\operatorname{Ei}(1, z)=\int_{z}^{\infty} \frac{\mathrm{e}^{t} d t}{t}
$$

The series expansion:

$$
\operatorname{Ei}(1, z)=-C-\ln z+z-z^{2} / 2+z^{3} / 18-\ldots,
$$

where $C$ is Euler constant, $\mathrm{e}^{C}=1.781072418$.

The asymptotic expansion:

$$
\operatorname{Ei}(1, z)=\frac{\mathrm{e}^{-z}}{z}\left(1-\frac{1}{z}+\frac{2}{z^{2}}+\ldots\right)
$$




\section{B. Probability integral}

The definition:

$$
\operatorname{erf}(z)=\frac{2}{\sqrt{\pi}} \int_{0}^{z} \mathrm{e}^{-t^{2}} d t
$$

The series expansion:

$$
\operatorname{erf}(z)=\frac{2 z}{\sqrt{\pi}}\left(1-z^{2} / 3+z^{4} / 10-\ldots\right)
$$

The asymptotic expansion:

$$
\operatorname{erfc}(z) \equiv 1-\operatorname{erf}(z)=\frac{\mathrm{e}^{-z^{2}}}{\sqrt{\pi} z}\left(1-\frac{1}{2 z^{2}}+\frac{3}{4 z^{2}}-\ldots\right)
$$

In Sec. 7 we used the following indefinite integral:

$$
\int \operatorname{erf}(\sqrt{z}) \frac{d z}{z}=4 \sqrt{\frac{z}{\pi}}{ }_{2} F_{2}\left(\frac{1}{2}, \frac{1}{2} ; \frac{3}{2}, \frac{3}{2} ;-z\right),
$$

where

$$
{ }_{2} F_{2}\left(\alpha_{1}, \alpha_{2} ; \beta_{1}, \beta_{2} ; x\right)=\sum_{k=0}^{\infty} \frac{\alpha_{1 k}, \alpha_{2 k}}{\beta_{1 k}, \beta_{2 k}} \frac{x^{k}}{k !}, \quad \alpha_{k}=\alpha(\alpha+1) \cdots(\alpha+k), \ldots
$$

is the generalized hypergeometric function. To obtain an asymptotic behavior of the latter for $z \rightarrow \infty$ we use the following identity:

$$
\int_{0}^{\mathbf{z}} \ln w \frac{\exp (-w)}{\sqrt{\pi w}} d w=\ln z \operatorname{erf}(\sqrt{z})-4 \sqrt{\frac{z}{\pi}}{ }_{2} F_{2}\left(\frac{1}{2}, \frac{1}{2} ; \frac{3}{2}, \frac{3}{2} ;-z\right),
$$

which can be easily proved integrating by parts. Then taking into account the integral

$$
\int_{0}^{\infty} \ln w \frac{\exp (-w)}{\sqrt{\pi w}} d w=-C-2 \ln 2,
$$

we find that for $z \rightarrow \infty$

$$
4 \sqrt{\frac{z}{\pi}}{ }_{2} F_{2}\left(\frac{1}{2}, \frac{1}{2} ; \frac{3}{2}, \frac{3}{2} ;-z\right) \approx \ln \left(4 z \mathrm{e}^{C}\right) .
$$

Integration of the functions $g(y)$ defined in (181) and $f_{2}(y)$ defined in (170) over $y$ can be performed analytically:

$$
\begin{aligned}
S_{1}(w) & =\int_{0}^{w} g(y) d y=300 \sqrt{\frac{w}{\pi}}\left[{ }_{2} F_{2}\left(-\frac{1}{2},-\frac{1}{2} ; \frac{1}{2}, \frac{3}{2} ;-w\right)-{ }_{2} F_{2}\left(-\frac{1}{2},-\frac{1}{2} ; \frac{1}{2}, \frac{1}{2} ;-w\right)\right] \\
& +75 w\left(1-\ln \left(4 w \mathrm{e}^{C}\right)\right)+\left(\frac{169}{2} w+\frac{2}{3} w^{4}+57 w^{2}-10 w^{3}-\frac{189}{8}\right) \operatorname{erfc}(\sqrt{w})- \\
& -\frac{1}{12} \frac{\mathrm{e}^{-y} \sqrt{y}}{\sqrt{\pi}}\left(8 w^{3}-124 w^{2}+750 w+567\right)+\frac{189}{8}, \\
S_{2}(w) & =\int_{0}^{w} f_{2}(y) d y=12 \sqrt{\frac{w}{\pi}}\left[{ }_{2} F_{2}\left(-\frac{1}{2},-\frac{1}{2} ; \frac{1}{2}, \frac{3}{2} ;-w\right)-{ }_{2} F_{2}\left(-\frac{1}{2},-\frac{1}{2} ; \frac{1}{2}, \frac{1}{2} ;-w\right)\right] \\
& +3 w\left(1-\ln \left(4 w \mathrm{e}^{C}\right)\right)+\frac{1}{24}\left(60 w-16 w^{4}+72 w^{2}-48 w^{3}-9\right) \operatorname{erfc}(\sqrt{w})+ \\
& +\frac{1}{24} \frac{\mathrm{e}^{-y} \sqrt{y}}{\sqrt{\pi}}\left(16 w^{3}+40 w^{2}-84 w-18\right)+\frac{3}{8} .
\end{aligned}
$$


For small arguments the leading terms are

$$
\begin{aligned}
& S_{1}(w) \simeq-75 w \ln w \\
& S_{2}(w) \simeq-3 w \ln w .
\end{aligned}
$$

[1] N. Jones, H. Stoica, S.-H. H. Tye, Brane Interaction as the Origin of Inflation, JHEP 0207, 051 (2002); The production, spectrum and evolution of cosmic strings in brane inflation, Phys. Lett. B B563, 6 (2003) arXiv:hep-th/0303269].

[2] S. Sarangi and S.-H. H. Tye, Cosmic string production towards the end of brane inflation, Phys. Lett. B 536, 185 (2002) arXiv:hep-th/0204074.

[3] G. R. Dvali and S.-H. H. Tye, Brane inflation, Phys. Lett. B 450, 72 (1999) arXiv:hep-ph/9812483.

[4] S. Kachru, R. Kallosh, A. Linde, J. Maldacena, L. McAllister and S. P. Trivedi, Towards inflation in string theory, JCAP 0310, 013 (2003) arXiv:hep-th/0308055.

[5] J.J. Blanco-Pillado, C.P. Burgess, J.M. Cline, C. Escoda, M. Gomez-Reino, R. Kallosh, A. Linde, F. Quevedo, Racetrack Inflation JHEP 0411 (2004) 063 [arXiv:hep-th/0406230].

[6] H. Firouzjahi and S.-H. H. Tye, Brane Inflation and Cosmic String Tension in Superstring Theory, JCAP 0503, 009 (2005) arXiv:hep-th/0501099].

[7] S. E. Shandera and S.-H. H. Tye, Observing Brane Inflation, JCAP 0605, 007 (2006) arXiv:hep-th/0601099.

[8] S. H. S. Alexander, Inflation from D - anti-D brane annihilation, Phys. Rev. D65, 023507 (2002) arXiv:hep-th/0105032]; C. P. Burgess, M. Majumdar, D. Nolte, F. Quevedo, G. Rajesh and R. J. Zhang, The inflationary brane-antibrane universe, JHEP 0107, 047 (2001) arXiv:hep-th/0105204].

[9] J. Polchinski, Cosmic string revisited, AIP. Conf. Proc. 743, 331 (2004); Int. J. Mod. Phys. A20, 3413 (2004) arXiv:hep-th/0410082].

[10] A. C. Davis and T. W. B. Kibble, Fundamental cosmic strings, Contemp. Phys. 46, 313 (2005) arXiv:hep-th/0505050.

[11] J.M. Cline, Inflation from String Theory, invited talk at JGRG14 conference, Kyoto, Japan, 2 Dec. 2004, arXiv:hep-th/0501179].

[12] A. Vilenkin, Cosmic strings: progress and problems, arXiv:hep-th/0508135].

[13] M. Sakellariadou, arXiv:hep-th/0602276].

[14] G. R. Dvali and A. Vilenkin, Formation and evolution of cosmic D strings, JCAP 0403, 010 (2004) [arXiv:hep-th/0312007].

[15] E. J. Copeland, R. C. Myers and J. Polchinski, Cosmic F and D strings, JHEP 0406, 013 (2004); Comptes Rendus Physique 5, 1021 (2004) arXiv:hep-th/0312067.

[16] J. Polchinski, Introduction to cosmic F- and D-strings, Cargese 2004, String theory: From gauge interactions to cosmology, 229-253, (2004).

[17] M. G. Jackson, N. T. Jones, J. Polchinski, Collisions of Cosmic F-and D-strings, JHEP 0510, 0132005 arXiv:hep-th/0405229].

[18] P. M. Saffin, JHEP 509, 011 (2005) [arXiv:hep-th/0506138].

[19] E. J. Copeland, T. W. B. Kibble, D. A. Steer, newblock Collisions of strings with Y junctions, arXiv:hep-th/0601153.

[20] M. Sakellariadou, A Note on evolution of cosmic string/superstring networks, JCAP 0504, 003 (2005) arXiv:hep-th/0410234].

[21] C. J. A. P. Martins, Scaling laws for non-intercommuting cosmic string networks, Phys. Rev. D 70, 107302 (2004) arXiv:hep-th/0410326].

[22] E. Copeland, P. Saffin, On evolution of cosmic-superstringnetworks, JHEP 0511, 023 (2005) [arXiv:hep-th/0505110].

[23] A. Avgoustidis, E.P.S. Shellard, Cosmic string evolution in higher dimensions, Phys. Rev. D 71, 123513 (2005) arXiv:hep-th/0512582].

[24] C. H. H. Tye, I. Wasserman and M. Wyman, Scaling of multi-tension cosmic string networks, Phys. Rev. D 71, 103508 (2005) arXiv:astro-ph/0503506.

[25] T. Vachaspati and A. Vilenkin, Gravitational radiation from cosmic strings, Phys. Rev. D 31, 3052 (1985).

[26] C. J. Burden, Gravitational radiation from a particularclasscosmic trings, Phys. Lett. B 164, 277 (1985).

[27] A. Vilenkin, Cosmic Strings And Domain Walls, Phys. Rep. 121, 263 (1985).

[28] D. Garfinkle and T. Vachaspati, Radiation from kinky, cuspless cosmic loops, Phys. Rev. D 36, 3339 (1987).

[29] R. L. Davis, Cosmic axions from cosmic strings, Phys. Lett. B 180, 225 (1985).

[30] A. Vilenkin, T. Vachaspati, Radiation of goldstone bosons from cosmic strings, Phys. Rev. D 35, 1138 (1985).

[31] R. L. Davis, Goldstone bosons in string models of galaxy formation, Phys. Rev. D 32, 3172 (1985).

[32] A. Dabholkar, J. Quashnock, Pinning down the axion, Nucl. Phys. B 333, 815 (1989).

[33] E.J. Copeland, D. Haws, and M. Hindmarsh, Classical theory of radiating strings, Phys. Rev. D 42, 276 (1990).

[34] D. Garfinkle and T. Vachaspati, Radiation of goldstone bosons from cosmic strings, Phys. Rev. D 36, 2229 (1987).

[35] T. Damour and A. Vilenkin, Cosmic string and the string dilaton, Phys. Rew. Lett. 78, 2288 (1997).

[36] E. Babichev and M. KachelrieB, Constraining cosmic superstrings with dilaton emission, Phys. Lett. B 614,1 (2005) arXiv:hep-th/0502135. 
[37] D. V. Gal'tsov, Yu. V. Grats, and P. S. Letelier, Post-linear formalism for gravitating strings: crossed straight strings collision, Ann. of Phys. 224, 90 (1993).

[38] J. L. Cornou, E. Pajer, R. Sturani, Graviton production from D-string recombination and annihilation, Nucl.Phys. B756, 16-37 (2006) arXiv:hep-th/0606275.

[39] D. V. Gal'tsov, Yu. V. Grats, and A. B. Lavrentiev, Cerenkov radiation from superconducting cosmic strings, JETP Lett. 59, 385 (1994).

[40] D. V. Gal'tsov, E. Yu. Melkumova and R. Kerner, Axion bremsstrahlung from collissions of global strings, Phys. Rev. D 70, 045009 (2004) [arXiv:astro-ph/0310718].

[41] B. Carter and R. A. Battye, Nondivergence of gravitational selfinteractions for Goto-Nambu strings, Phys. Lett. B430, 439 (1998) arXiv:hep-th/9803012.

[42] A. Buonanno, T. Damour, Effective action and tension renormalisation for cosmic and fundamental strings, Phys. Lett. B 432, 51 (1998) arXiv:hep-th/9803025.

[43] R. A. Battye, E. P. S. Shellard, Radiative back reaction on global strings, Phys. Rev. D53, 19961811 [arXive hep-ph/9508301].

[44] B. Carter, Electromagnetic selfinteraction in strings, Phys. Lett. B404, 246 (1997) |arXiv:hep-th/9704210|.

[45] B. Carter, Renormalization of gravitational self-interaction for wiggly strings, Phys. Rev. D60, 083502 (1999) [arXiv:hep-th/9806206].

[46] Yu. V. Grats, A.A. Rossikhin and A.O. Sboichakov, Chiral string in a curved space: gravitational self-force Mod. Phys. Lett. A16, 725-7300 (2001) |arXiv:hep-th/9806206];

Gravitation and Cosmology, 7, 178-182 (2001). 